\title{
Isolation by Distance in Populations with Long-Range Dispersal
}

Tyler B. Smith and Daniel B. Weissman

Department of Physics, Emory University, Atlanta, Georgia 30322

\begin{abstract}
Limited dispersal results in isolation by distance in spatially structured populations, in which individuals found further apart tend to be less related to each other. Models of populations undergoing short-range dispersal predict a close relation between the distance individuals disperse and the length scale over which two sampled individuals are likely to be closely related. In this work, we study the effect of long jumps on patterns of isolation by distance by replacing the typical short-range dispersal kernel with a long-range, power-law kernel. We find that incorporating long jumps leads to a slower decay of relatedness with distance, and that the quantitative form of this slow decay contains visible signatures of the underlying dispersal process.
\end{abstract}

KEYWORDS Isolation By Distance; Identity By Descent; Dispersal Rate; Long-Range Dispersal

\section{Introduction}

Direct measurement of dispersal in natural populations is often difficult or impossible due to practical difficulties in tracking large numbers of individuals over long periods of time. It is often more feasible to instead infer dispersal from spatial patterns of genetic diversity (Cayuela et al. 2018; Bradburd and Ralph 2019; Battey et al. 2020). Populations with limited dispersal should exhibit "isolation by distance": the more distant individuals are from each other in space, the less related they tend to be (Wright 1946; Rohlf and Schnell 1971; Slatkin 1991). While in general the spatial pattern of genetic diversity depends on selection (Barton et al. 2013; Allman and Weissman 2018), for populations evolving neutrally the strength of isolation by distance is simply determined by the balance between dispersal and mutation, and thus if the mutation rate is known, the dispersal rate can be inferred directly (Malécot 1975; Slatkin and Arter 1991; Slatkin 1993).

For populations spread over a fairly continuous range, rather than being clumped into a small number of discrete subpopulations, dispersal is often assumed to be short-range, with displacement approximately following a normal distribution (Barton et al. 2002; Ringbauer et al. 2017). If dispersal is unbiased and homogeneous, it is then characterized by a single parameter, the dispersal rate $D$ (the diffusion constant). Pairwise genetic similarity is predicted to decay exponentially with distance, with a decay rate of $\sqrt{\mu / D}$, where $\mu$ is the mutation rate (Kimura and Weiss 1964; Slatkin and Barton 1989; Rousset 1997, 2000; Barton et al. 2002; Ralph and Coop 2013).
However, many populations exhibit at least occasional longrange dispersal (Clark 1998; Willson 1993; Atkinson et al. 2002; Baguette 2003; Brockmann et al. 2006; Dai et al. 2007; Fric and Konvicka 2007; Devaux et al. 2007; Aguillon et al. 2017; Vallaeys et al. 2017). For many other populations, particularly non-animal ones, very little is known about dispersal, and we would like to know how to use genetic data to determine if it is short- or long-range (Nathan et al. 2003; Cayuela et al. 2018). Even for populations where dispersal is primarily short-range, even very rare long jumps that are unlikely to be observed directly (Koenig et al. 1996) can have large effects on the population's evolution (Ibrahim et al. 1996; Mancinelli et al. 2003; Bialozyt et al. 2006; Brockmann and Hufnagel 2007; Wingen et al. 2007; Fayard et al. 2009; Hallatschek and Fisher 2014; Paulose et al. 2019; Paulose and Hallatschek 2020), so we would also like to be able to infer their pattern and frequency.

In this work, we further explore the effects of long-range (power-law) dispersal in neutrally evolving, demographically stable populations with constant density. This problem was previously studied by Nagylaki (1976) in one dimension for moderately long-range dispersal with finite mean distance and by Chave and Leigh Jr (2002) in two dimensions for Cauchydistributed dispersal. Recently, Janakiraman (2017) studied an analogous problem in chemical physics in the same regime as Nagylaki (1976) and found complementary results. We unify and extend this work to cover arbitrary power law dispersal tails in both one and two spatial dimensions, and find expressions for how the pattern of isolation by distance reflects the underlying dispersal process at both large and small distances. We also find 


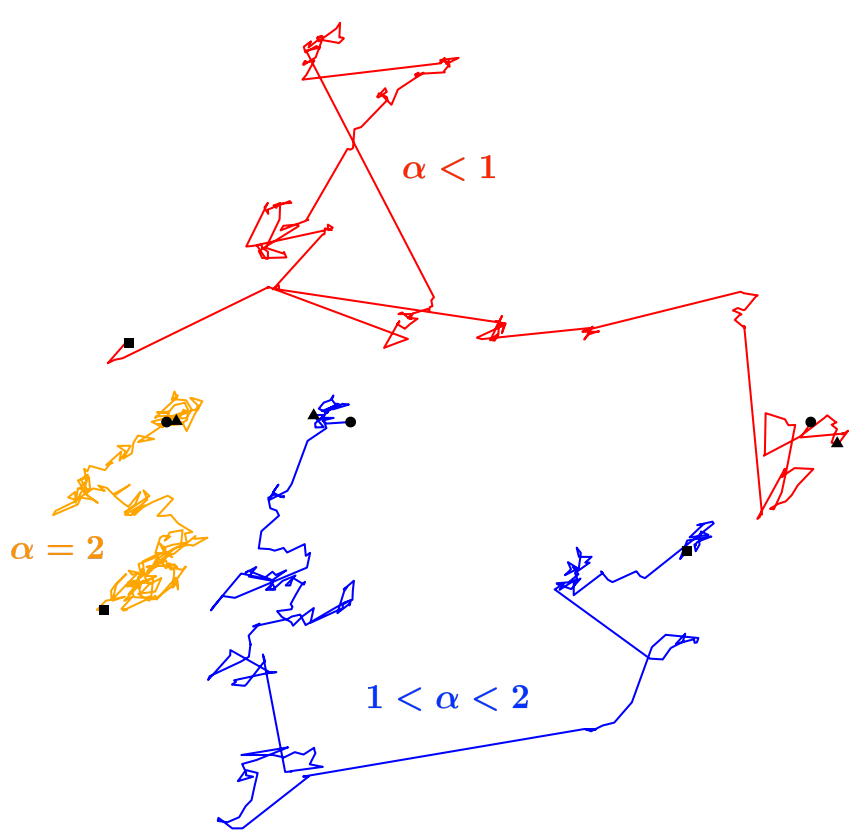

Figure $1 \alpha$ controls the size and number of long-range

jumps. Two-dimensional Lévy flights with $\alpha$ values $0.95,1.5$, and 2. When $\alpha=2$, Lévy flight motion reduces to normal diffusion without any long-range jumps. Lévy flights with $\alpha<2$ have divergent mean squared displacement and a power law dispersal kernel proportional to $x^{-\alpha-1}$. For $\alpha<1$, the mean displacement also diverges, and large jumps become noticeably more prevalent than for $1<\alpha<2$. Circles mark the beginnings of the trajectories, while squares mark the ends. The generalized dispersal constants $D_{\alpha}$ are chosen such that the flights all have the same characteristic displacement at the time step marked by triangles. On shorter time scales, the diffusive trajectory tends to have the largest displacement, while on longer time scales the trajectory with $\alpha<1$ tends to have the largest displacement.

how the distribution of time to the most recent common ancestor of a pair of individuals depends on the distance between them. Forien (2019) recently proposed a similar model for populations evolving according to a spatial $\Lambda$-Fleming-Viot process with long-range dispersal; our analysis may also apply to this model.

\begin{tabular}{cl}
\hline Symbol & Definition \\
$x$ & Distance between samples \\
$\rho$ & Population density \\
$\alpha$ & Stability parameter of dispersal tail \\
\hline$D_{\alpha}$ & Generalized dispersal constant \\
$\mu$ & Mutation rate \\
$\psi$ & Probability of identity \\
$\bar{x}=\left(D_{\alpha} / \mu\right)^{1 / \alpha}$ & Characteristic length scale of identity
\end{tabular}

alpha-stable distribution:

$$
K_{1}(y \mid t)=\frac{1}{2 \pi} \int_{-\infty}^{\infty} d k \exp \left(-i k y-D_{\alpha} t|k|^{\alpha}\right) .
$$

The parameter $D_{\alpha}$ is a generalized diffusion constant with units of length ${ }^{\alpha} /$ time. It sets the scale of dispersal: at time $t$, a typical lineage will have a displacement $\bar{y}(t) \sim\left(D_{\alpha} t\right)^{1 / \alpha}$. As shown in Fig. 1, the "stability parameter" $\alpha, 0<\alpha \leq 2$, controls the tail of dispersal: for $\alpha<2$, the probability that at time $t$ a lineage has moved an abnormally long distance $y \gg \bar{y}(t)$ is $K_{1}(y \mid t) \propto y^{-1-\alpha}$, a power law tail. In the limiting case $\alpha=2$, dispersal reduces to ordinary short-range diffusion and $K_{1}$ is just a normal distribution with standard deviation $\sqrt{D t}$. In two dimensions, the definition (1) must be adjusted (see (43) in the Methods), but the only change to the approximate description above is that the tail is $\propto y^{-2-\alpha}$.

When the two lineages encounter each other, they coalesce at rate $1 / \rho$, where $\rho$ is the density of the population. Technically, for $\alpha \leq 1$ in one dimension the two lineages will never be at exactly the same position. In two dimensions, all values of $\alpha$ have this problem, including ordinary diffusion (Mörters and Peres 2010). So really there must be some small distance $\delta$ within which lineages coalesce at rate $\sim 1 /\left(\delta^{d} \rho\right)$, where $d$ is the dimensionality of the range. At these small scales, even the model of independent diffusion of lineages breaks down (Barton et al. 2010). But we will see below that this coalescence length scale does not affect isolation by distance on larger scales $x \gg \delta$.

We are interested in the probability $\psi$ of identity by descent of our sample pair as a function of the distance between them, $x$, which we will also refer to as the homozygosity or relatedness. If the time to their most recent common ancestor is $T$ and the mutation rate is $\mu$, then $\psi$ is given by:

$$
\psi(x)=E\left[e^{-2 \mu T} \mid x\right] .
$$

Although usually it is $\psi$ rather than the coalescence time $T$ itself that is directly observable, $T$ is important for, e.g., determining whether it is reasonable to assume stable demography, so we will also find expressions for its distribution $p(t \mid x)$.

\section{Results}

In this section, we will describe our main results and provide brief sketches of the logic behind key features. Roughly speaking, the basic intuition is that the sampled pair will be identical if their lineages coalesce within the past $\sim 1 / \mu$ generations. In this time, they will disperse a typical distance $\sim \bar{x} \equiv\left(D_{\alpha} / \mu\right)^{1 / \alpha}$, so this is the key length scale over which identity decays: pairs separated by $x \ll \bar{x}$ should be relatively closely related, while identity between pairs separated by $x \gg \bar{x}$ should be rare. 


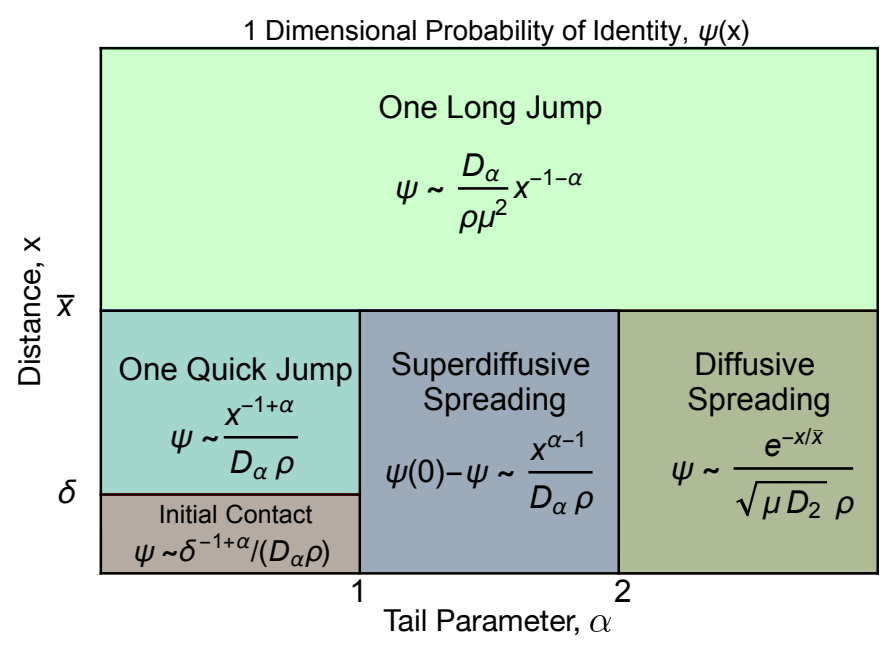

Figure 2 The form of isolation by distance in one dimension is universal at long distances and varies at short distance. Approximate form for the probability of identity as a function of distance, $\psi(x)$, for different dispersal kernels $\alpha$. Different regimes of the parameter space are separated by solid lines, and labelled by their qualitative dynamics. Coalescence for distant pairs, $x \gg \bar{x}$, where $\bar{x}=\left(D_{\alpha} / \mu\right)^{1 / \alpha}$ is the characteristic length scale of identity, occurs via one long jump for all $\alpha$, leading to the power law scaling at large distances predicted by (4). Coalescence for nearby pairs, $x \ll \bar{x}$, depends on the value of $\alpha$ considered. For $\alpha>2$, the motion of lineages across short distances is diffusive and $\psi$ scales exponentially, as shown in (3). For $1<\alpha<2$, short distances are covered via many small jumps, but lineages spread faster than they would under diffusion, leading to the broader scaling found in (5). For $\alpha<1$, even short distances are covered by one quick jump, leading to the power law shown in (6). Lineages that do not coalesce quickly (at $t \ll 1 / \mu$ ) will likely never coalesce, and probability of identity is limited by $\delta$, rather than $\mu$, as shown in (7). We use " $\sim$ " to denote proportionality in the limit of large population density where $\psi(0) \ll 1$.

For the classic case of diffusive motion $(\alpha=2)$, this length scale is $\bar{x}=\sqrt{D / \mu}$, and the probability of identity falls off exponentially (Barton et al. 2002):

$$
\psi(x)=\frac{e^{-x / \bar{x}}}{4 \rho \bar{x} \mu+1} .
$$

Here we generalize (3) to $\alpha \neq 2$, and find simple approximate expressions for $\psi$ in different parameter regimes, illustrated in Fig. 2, including a universal form for all dispersal kernels at long distances. Intuitively, long-range dispersal broadens the distribution of coalescence times for pairs at a given separation $x$, creating more overlap in the distributions for different $x$ values (Fig. 3). must then coalesce within their neighborhood of $\sim \delta \rho$ individuals before they mutate, which occurs with probability $\sim 1 /(\mu \rho \delta)$. We therefore expect that the probability of identity is $\psi \sim D_{\alpha} x^{-\alpha-1} \delta / \mu /(\mu \rho \delta)=D_{\alpha} x^{-\alpha-1} /\left(\mu^{2} \rho\right)$, i.e., that there is a power-law dependence of identity on distance, with the same exponent as that of dispersal. A more careful calculation (see Methods) recovers the result Eq. (37) of Nagylaki (1976):

$$
\psi(x \gg \bar{x}) \approx \frac{\Gamma(1+\alpha) \sin (\pi \alpha / 2)}{2 \pi} \frac{D_{\alpha}}{\mu^{2} \rho x^{1+\alpha}},
$$

up to an additional prefactor that enters when $\psi(0)$ is close to one. We confirm (4) with simulations and numerical analysis (Fig. 4).

\section{Nearby pairs: moderately long-range dispersal}

For moderately long-rage dispersal, $1<\alpha \leq 2$, nearby pairs should be nearly as related as pairs from the same deme: $\psi(x \ll \bar{x}) \approx \psi(0)$. In the time $\sim 1 / \mu$ before they mutate, the lineages will disperse over a distance $\sim \bar{x}$. There are $\sim \rho \bar{x}$ individuals in this neighborhood, so the overall average rate of coalescence is $\sim 1 /(\rho \bar{x})$. If this is large compared to the mutation rate, then homozygosity will be high, $\psi(0) \approx 1$, while if coalescence is slow compared to mutation, homozygosity will be low, $\psi(0) \sim 1 /(\mu \rho \bar{x})$. A more detailed analysis (see Methods) recovers the result of Nagylaki (1976) (Eq. (39)) for $\psi(0)$, and the leading distance dependence was found by Janakiraman (2017) (Eq. (C1)):

$$
\psi(x \ll \bar{x}) \approx \frac{1-\frac{\alpha \sin (\pi / \alpha)}{\Gamma(\alpha) \cos (\pi(1-\alpha / 2))}\left(\frac{x}{\bar{x}}\right)^{\alpha-1}}{2 \alpha \sin (\pi / \alpha) \rho \bar{x} \mu+1} .
$$

We confirm (5) with simulations and numerical analysis (Fig. 4). The scaling in (5) reflects the superdiffusive dispersal of the lineages.

\section{Nearby pairs: very long-range dispersal}

For very long-range dispersal in which the mean jump size diverges, $\alpha<1$, nearby lineages are likely to either coalesce very quickly or to disperse across the whole range before coalescing (Palyulin et al. 2014). This "now-or-never" dynamic has the interesting effect of making the local probability of identity by descent independent of the mutation rate (Fig. 5), since the main competition is between coalescence and long-range dispersal rather than between coalescence and mutation. Intuitively, the pair of lineages take time $t_{0} \sim x^{\alpha} / D_{\alpha}$ to disperse across the distance between them. From that time on, they are roughly evenly spread over a range $\sim\left(D_{\alpha} t\right)^{1 / \alpha}$, and so coalesce at rate $\sim\left(D_{\alpha} t\right)^{-1 / \alpha} / \rho$. Integrating this rate over time starting from $t_{0}$ out to $\sim 1 / \mu$, we find that $\psi \sim 1 /\left(\rho D_{\alpha} x^{1-\alpha}\right)$, with the upper limit of integration only negligibly decreasing $\psi$. So $\psi$ again follows a power law, although a different one from the longdistance $1 / x^{1+\alpha}$. We calculate $\psi$ more carefully in the Methods to find:

$$
\psi(\delta \ll x \ll \bar{x}) \approx \frac{\Gamma(1-\alpha) \sin (\pi \alpha / 2)}{2 \pi} \frac{1}{\rho D_{\alpha} x^{1-\alpha}},
$$

again up to an additional prefactor that enters when $\psi(0)$ is close to one. We confirm (6) with simulations and numerical analysis (Fig. 4 and Fig. 5).

The power law in (6) makes it diverge at very short distances, where it breaks down. Instead, for individuals within the same deme, $x<\delta, \psi$ flattens out. Roughly speaking, individuals 


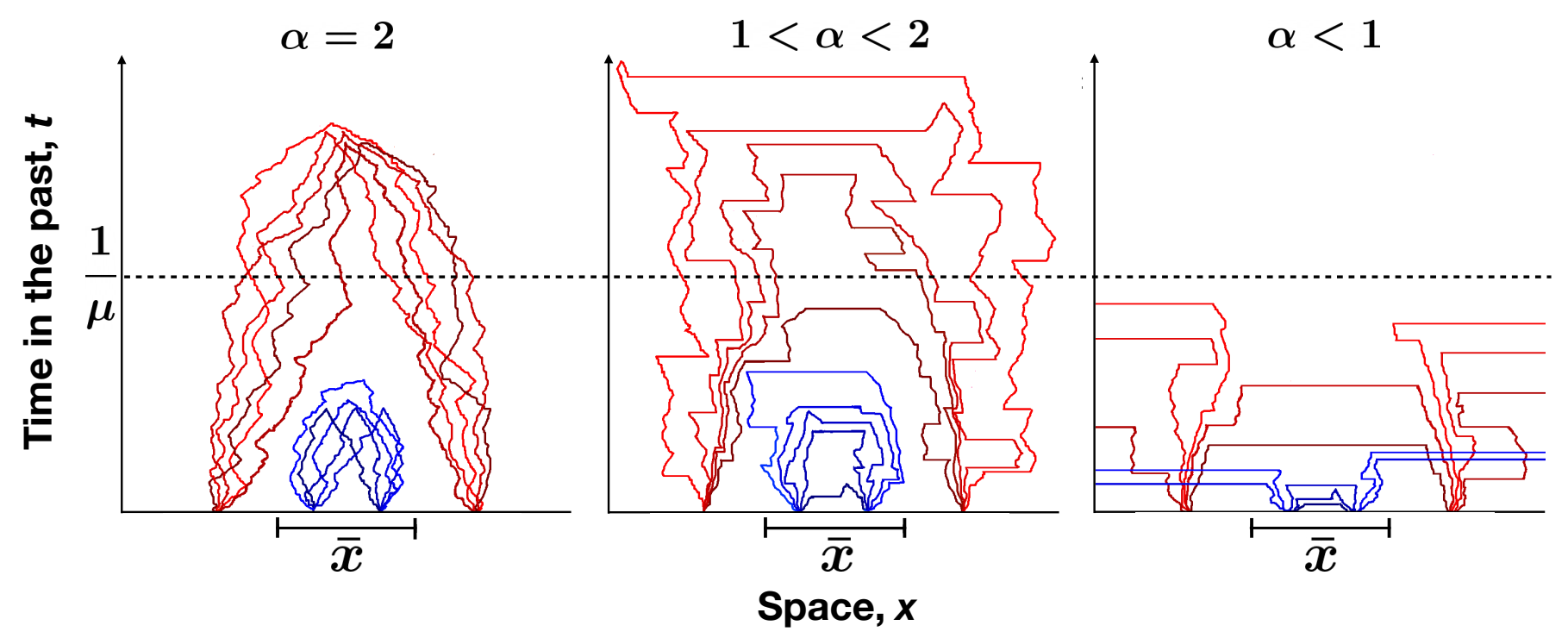

Figure 3 Long-range jumps affect when and where lineages coalesce. Qualitative illustrations of lineage dynamics for each of the three $\alpha$ regimes in 1 dimension. Typical histories are shown for nearby samples $(x \ll \bar{x}$, blue) and distant samples $(x \gg \bar{x}$, red). Left: For diffusive motion, $\alpha=2$, initial separation $x$ is a relatively good predictor of coalescence time. Center: For moderately long-range dispersal, $1<\alpha<2$, large jumps broaden the spatial and temporal ranges over which lineages coalesce. Lineages at large separations $x \gg \bar{x}$ are occasionally able to coalesce at times comparable to $1 / \mu$. Right: For very long-range dispersal, $\alpha<1$, large jumps are common. This allows for rapid coalescence of lineages at both small and large distances, but also lets lineages jump very far away from each other and avoid coalescing.

coalescence at rate $1 /(\rho \delta)$ and disperse outside of coalescence range at rate $\sim D_{\alpha} \delta^{-\alpha}$. When coalescence is faster, probability of identity is high, $\psi(0) \approx 1$, while when dispersal is faster it is low, $\psi \sim 1 /(\rho \delta) /\left(D_{\alpha} \delta^{-\alpha}\right)=1 /\left(\rho D_{\alpha} \delta^{1-\alpha}\right)$. A more careful calculation gives (see Methods):

$$
\psi(x \ll \delta) \approx 1 /\left[1+\frac{2^{(\alpha+3) / 2} \pi}{\Gamma(1 / 2-\alpha / 2)} \rho D_{\alpha} \delta^{1-\alpha}\right],
$$

although these numerical factors depend on the details of the model and are only valid when $\psi(0)$ is small. We confirm (7) with simulations (Fig. 5).

\section{Finite Variance Dispersal}

In addition to considering Lévy flight dispersal kernels with $0<\alpha \leq 2$, we consider F-distribution kernels (see Methods) with steeper power law tails $\alpha>2$. These have finite variance and approach a diffusion after infinitely many steps, but at any finite time will be different, particularly in the tail. As shown in Fig. 6 , for nearby pairs, $x \ll \bar{x}$, relatedness decays exponentially, as in the case of purely diffusive motion. However, for distant pairs, $x \gg \bar{x}$, coalescence tends to occur via one long jump, and the probability of identity follows the power law jump tail $\propto x^{-\alpha-1}$ (Fig. 6), i.e., the same behavior as the infinite-variance Lévy flights described above.

\section{Two Dimensions}

While we have focused above on the case of one spatial dimension, the same intuition applies in two dimensions. In some ways the results are even simpler-the same expressions apply for both $\alpha<1$ and $1<\alpha<2$ (Fig. 7). For distant individuals, $x \gg \bar{x}$, the dynamics of coalescence are again dominated by large jumps in a single generation, and the probability of identity by descent again has the same power law tail as the dispersal kernel, which is now $\alpha+2$ :

$$
\psi(x \gg \bar{x}) \approx \sin \left(\frac{\pi \alpha}{2}\right) \frac{2^{\alpha-1} \Gamma(1+\alpha / 2)^{2}}{\pi^{2}} \frac{D_{\alpha}}{\rho \mu^{2} x^{2+\alpha}} .
$$

For nearby individuals, $\delta \ll x \ll \bar{x}$, all values of $\alpha<2$ display "now-or-never" behavior similar to that of $\alpha<1$ in one dimension. Coalescence tends to occur via one "quick jump", rather than many small jumps, and the competition is with jumping very far away rather than with mutating. The same intuitive argument used above applies, now with the lineages spread over an area of $\sim\left(D_{\alpha} t\right)^{2 / \alpha}$ and therefore coalescing at rate $\sim\left(D_{\alpha} t\right)^{-2 / \alpha} / \rho$, for a probability of identity $\psi \sim 1 /\left(\rho D_{\alpha} x^{2-\alpha}\right)$, independent of the mutation rate. We calculate this more carefully in the Methods, finding:

$$
\psi(\delta \ll x \ll \bar{x}) \approx \frac{\Gamma(1-\alpha / 2)}{2^{\alpha} \pi \Gamma(\alpha / 2)} \frac{1}{\rho D_{\alpha} x^{2-\alpha}} .
$$

Note that (9) reduces to Eq. (A6) in Chave and Leigh Jr (2002) when $\alpha=1$.

Again, (9) must be cut off at very small distances, $x \lesssim \delta$, at which point homozygosity flattens out to its $x=0$ value:

$$
\psi(x \ll \delta) \approx 1 /\left[1+\frac{2^{2+\alpha / 2} \pi}{\Gamma(1-\alpha / 2)} \rho D_{\alpha} \delta^{2-\alpha}\right] .
$$

Just as in one dimension, the numerical factors in (10) depend on the details of the model and are only valid when $\psi(0) \ll 1$, but the basic scaling is general and arises from the race between coalescence at rate $\sim 1 /\left(\rho \delta^{2}\right)$ and dispersing outside coalescence range at rate $\sim D_{\alpha} \delta^{-\alpha}$. 

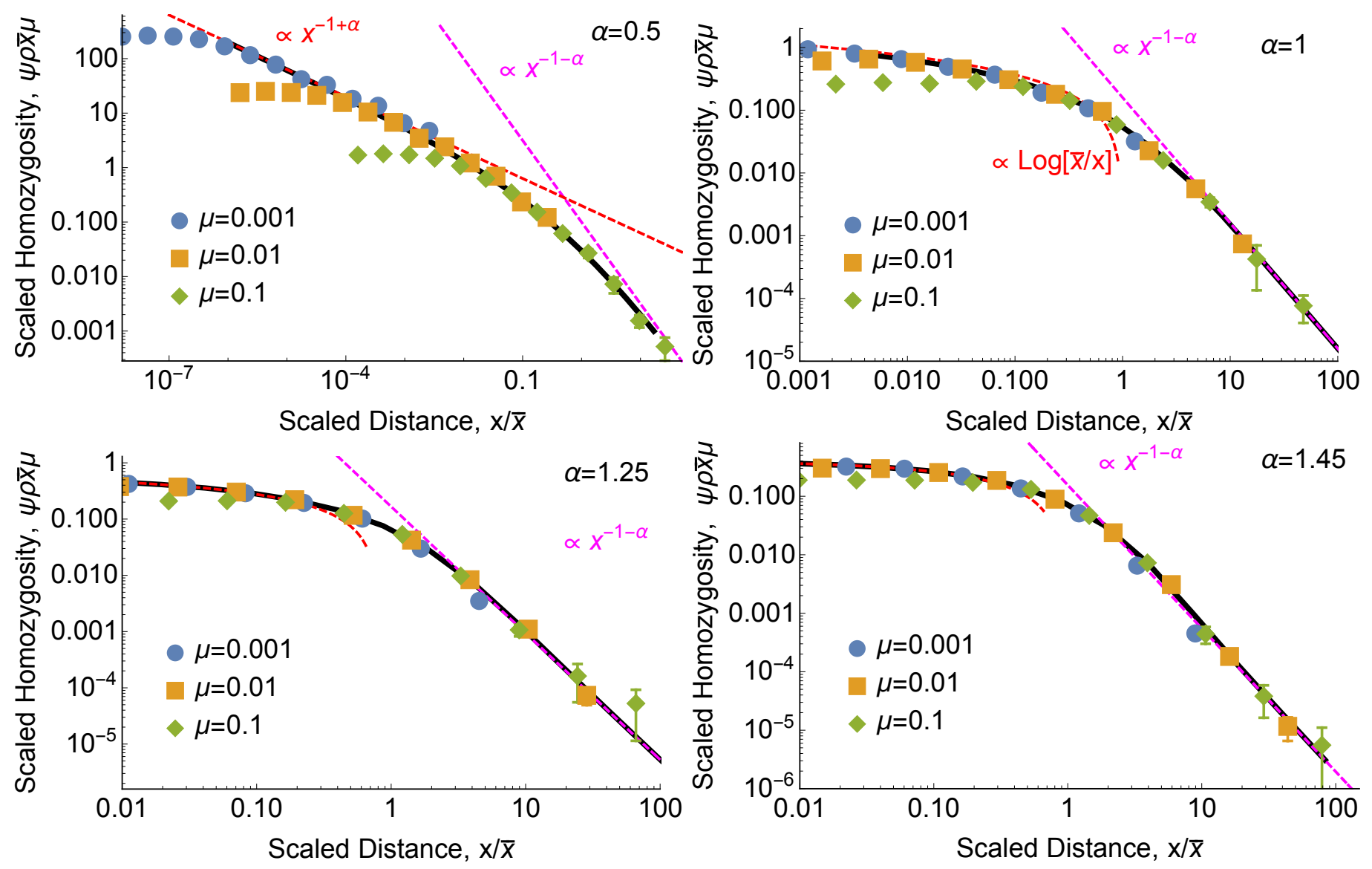

Figure 4 Isolation by distance follows the same power law as dispersal. Each panel shows the scaled probability of identity between a sampled pair of individuals, $\psi \rho \bar{x} \mu$, as a function of the scaled distance $x / \bar{x}$ between them. Points show simulation results, black curves show numerical solutions of $\psi(x)$ calculated from (27) with $\delta=0$, and magenta lines show the power law that emerges at large distances (4). Red curves show the asymptotic behavior predicted at short distances by (6) $(\alpha<1)$, (5) $(1<\alpha<2)$, and (40) $(\alpha=1)$. For all plots, error bars show $68 \%$ percentile bootstrap confidence intervals (see Methods). $\rho=100$ in all plots, and data with $\rho=10$ and $\rho=1$ (not shown) yield indistinguishable plots. 


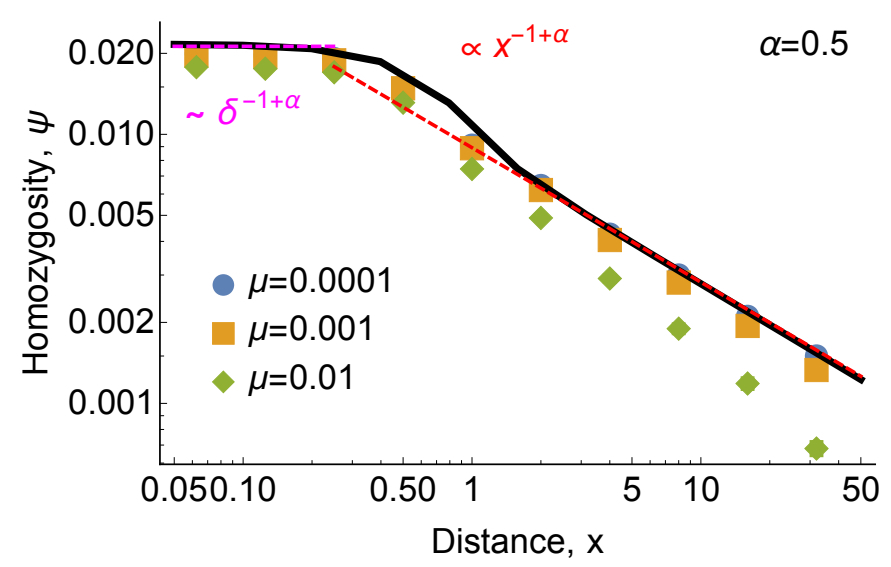

Figure 5 For very long-range dispersal, $\alpha<1$, relatedness at short distances is independent of mutation rate. Nearby lineages at $x \ll \bar{x}$ either coalesce quickly and are identical, or jump very far away from each other and never coalesce. Points show simulation results, and red and magenta lines show the asymptotic predictions of (6) and (7), respectively. The black curve shows a numerical solution of $\psi(x)$ calculated from (27) with $\mu=10^{-4} \cdot \rho=100$ in all plots, and data with $\rho=10$ and $\rho=1$ (not shown) yield indistinguishable plots.

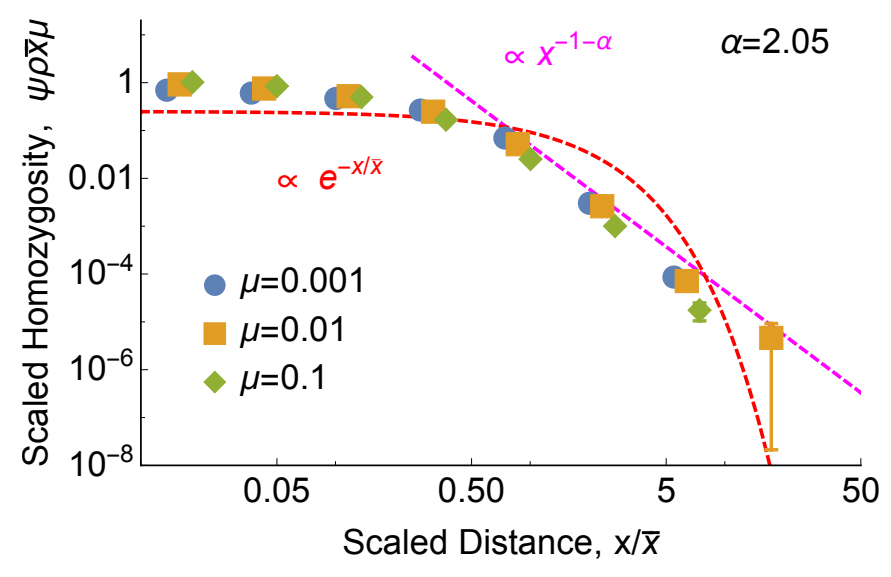

Figure 6 Even for $\alpha>2$, relatedness still follows the same power law as dispersal, rather than the diffusive prediction. Points show simulation results with $\rho=100 ; \rho=10$ and $\rho=1$ yield indistinguishable plots. Since the dispersal kernel has finite variance, it approaches a diffusion, and at short distances $x \ll \bar{x}$ the probability of identity is well-approximated by the diffusive prediction (3) (red curve). But at long distances $x \gg \bar{x}$, relatedness is driven by rare long-range jumps, and therefore has the same power-law tail as dispersal, (31) (magenta line).

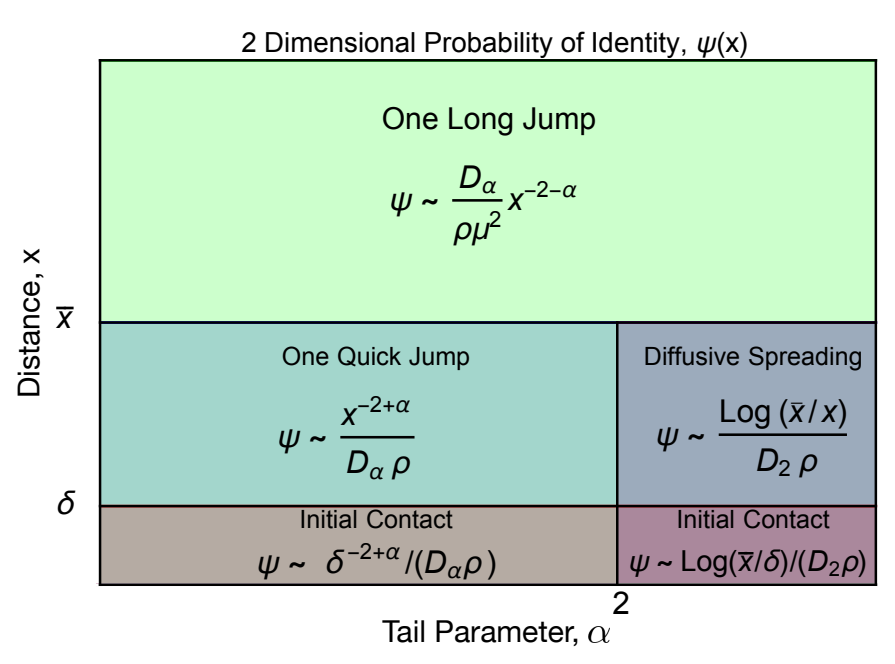

Figure 7 The form of isolation by distance in two dimensions is universal at long distances. Approximate form for the probability of identity as a function of distance, $\psi(x)$, for different dispersal kernels $\alpha$. Different regimes of the parameter space are separated by solid lines, and labelled by their qualitative dynamics. Coalescence for distant pairs, $x \gg \bar{x}$, typically occurs via one long jump, which leads to the power law scaling at large distances predicted by (8). Nearby pairs, $x \ll \bar{x}$, typically either coalesce very quickly or disperse far away from each other, so the probability of identity is nearly independent of the mutation rate, as shown in (9). This quick coalescence is effectively diffusive for $\alpha>2$, while for $\alpha<2$, it is typically driven by a single jump. We use " $\sim$ " to denote proportionality in the limit of large population density where $\psi(0) \ll 1$. 


\section{Discussion}

Limited dispersal produces a correlation between spatial and genetic distance (Wright 1946; Malécot 1975; Slatkin 1991; Slatkin and Arter 1991; Slatkin 1993). While most previous models have only considered diffusive dispersal, dispersal can be long-range in many natural populations. Nagylaki (1976) was the first to generalize classic diffusive models of isolation by distance by allowing dispersal distance to have a power law tail (with $1<$ $\alpha<2$ ). This groundbreaking work has largely been neglected; it was last cited by Chave and Leigh Jr (2002), who extended the results to two dimensions for the special case of Cauchy flights $(\alpha=1)$ in a paper modeling ecological diversity. We hope that this paper will reintroduce these results to population genetics now that the field may have sufficient data to apply them. We also extend this previous work by considering Lévy flights for all $\alpha \leq 2$ in both one and two dimensions, as well as steeper power law kernels $(\alpha>2)$ with finite variance. We find that, for all $\alpha$, long-range dispersal leads to much more long-range relatedness than diffusive dispersal, with relatedness having the same power law tail in distance as the dispersal kernel. This is true even for steep kernels with finite variance. In this case, even though a diffusive approximation can fit the pattern of isolation by distance between nearby individuals, it will greatly underestimate the degree of relatedness between distant individuals.

Standard methods for inferring dispersal from pairwise measures of relatedness or autocorrelations in allele frequency typically assume either short-range, diffusive motion (Rousset 1997, 2000; Robledo-Arnuncio and Rousset 2010; Ringbauer et al. 2017; Bradburd et al. 2018) (perhaps with recent long-range admixture (Bradburd et al. 2016)) or a small number of discrete demes (Slatkin 1991; Whitlock and McCauley 1999; Rousset and Leblois 2011; Petkova et al. 2016; Al-Asadi et al. 2019; Lundgren and Ralph 2019). Methods using cline theory to infer dispersal from the width of hybrid zones make similar assumptions about the motion of lineages being short-range and diffusive (Barton 1983; Barton and Hewitt 1985; Sotka and Palumbi 2006; Rieux et al. 2013; Gagnaire et al. 2015; Cayuela et al. 2018), and methods for non-stable demographies based on historical biogeography or coalescent theory tend to also assume a small number of discrete demes (Sanmartín et al. 2008; Ree and Smith 2008; Hey 2010). Other genetic methods such as parentage analysis are better equipped to infer long-range dispersal on continuous ranges, but these techniques require exhaustive sampling of the population to ensure that the parents of each individual can be located (Adams et al. 1992; Jones and Ardren 2003; Bacles and Ennos 2008; Wang and Santure 2009). More recent methods for pollen dispersal have been developed that allow for the inference of long-range dispersal without the need for exhaustive sampling, but knowledge of maternal genotypes for all sampled individuals is still required (Austerlitz et al. 2004; Robledo-Arnuncio et al. 2006). For plant species where this data is available, our results could serve as the basis for complementary inference methods. While the pollen dispersal methods are focused on inferring the dispersal kernel over a single generation, isolation by distance reflects the history of dispersal over many generations, so a comparison of the results could reveal changes in dispersal over time.

One key open question is to what extent it is possible to detect the genetic traces of rare long-range dispersal in natural populations, and if so how well the form of long-range dispersal (e.g., the tail exponent $\alpha$ ) can be determined. Austerlitz et al.
(2004) were able to detect long-range pollen dispersal in tree populations with the seed-specific TwoGener method, which suggests that similar patterns should be detectable from the genomes of the trees themselves. Aguillon et al. (2017) found clear patterns of isolation by distance across scales between 500 meters and 10 kilometers in Florida Scrub-Jays, and directly measured long-range dispersal; a good first test of an inference method would be to apply it to such a dataset to see if it can recover the known dispersal pattern.

Along with predicting characteristic scaling of identity by descent with distance, our results predict characteristic scalings with the mutation rate $\mu$, and also a scaling of the typical length scale of identity $\bar{x}$ with $\mu$. While mutation rate cannot be scanned directly as distance can, $\mu$ here should be understood as referring to the mutation rate in a block of non-recombining genome, and so a wide range of $\mu$ values can be scanned by considering identity by descent in blocks of varying size (Weissman and Hallatschek 2017). This will be valid as long as recombination is rare relative to mutation, or even if recombination is frequent as long as " $\mu$ " is understood to mean the sum of the block mutation and recombination rates. This suggests that it should be possible to measure identity by descent statistics corresponding to " $\mu$ " values ranging over five orders of magnitude in a single sample (Harris and Nielsen 2013).

Our analysis has focused on the lineages of a particular pair of individuals sampled a fixed distance apart in an infinitely large habitat. We can also consider a pair of individuals sampled from random locations from a habitat with finite length $L$; the mean coalescence time between them would then be the "effective population size". The pair will typically be sampled a distance $\sim L$ from each other, and so it will typically take a time $\sim L^{\alpha} / D_{\alpha}$ for their lineages to overlap in space. At this point the ancestry is effectively well-mixed, and it takes time proportional to the total population size $\sim L^{d} \rho$, where $d=1$ or 2 is the dimension of the habitat. For $L^{\alpha} / D_{\alpha} \ll L^{d} \rho$, the mixing time has little effect, while for $L^{\alpha} / D_{\alpha} \gtrsim L^{d} \rho$ spatial structure substantially increases genetic diversity. For short-range dispersal, $\alpha=2$, structure will be strong in a one dimensional habitat of length $L \gtrsim D \rho$ (Maruyama 1971), while in two dimensions its strength depends only on the local neighborhood size $D \rho$ (Maruyama 1972). The amount of population structure thus either increases with the spatial extent of the population (at fixed density) or is insensitive to it. With long-range dispersal, however, we see a new qualitative pattern. For $\alpha<d$, i.e., for any amount of long-range dispersal in two dimensions, the effect of structure on overall diversity counterintuitively becomes weaker as the range size $L$ grows, because $L^{\alpha} / D_{\alpha}$ grows more slowly than $L^{d} \rho$. It seems plausible that in many natural populations, spatial structure may be strong over short time scales (as measured by $\psi(x)$ at large $\mu$ ) while having little effect on the average coalescence time.

Our use of stable distributions for the dispersal kernel has been partly motivated by the fact that any isotropic singlegeneration dispersal kernel will converge to a stable one if it is repeated over many independent generations. But as we have noted, this is only true asymptotically, and in any real population there will be correlations across generations, spatial inhomogeneities, shifts in dispersal over time, limits due to finite range size, and many other effects that cannot be captured by a stable distribution. It is therefore better to see it as a simple null model, one step closer to reality than the purely diffusive one, that can serve as a background against which to measure 
all these other effects.

What other processes could produce similar patterns to longrange dispersal? One obvious one is if individuals are performing something more like a "Lévy walk" than a Lèvy flight, in which dispersal in any one generation is short but can be correlated across many generations (Zaburdaev et al. 2015). Such an effect can be produced at the level of alleles by hitchhiking on beneficial substitutions (Allman and Weissman 2018). But this should be readily distinguishable from neutral long-range dispersal by considering the distribution of relatedness across multiple individuals and loci - hitchhiking will produce longrange relatedness at the same few loci across all individuals, whereas neutral effects will be more evenly distributed. It is an open question whether other neutral processes, in particular demographic fluctuations, might produce similar patterns.

\section{Methods}

\section{Simulation Methods}

All simulation code and displayed data are available at https: //github.com/weissmanlab/Long_Range_Dispersal. We simulate our model in two stages. First, for each value of present-day separation $x$, dispersal constant $D_{\alpha}$, and tail parameter $\alpha$, we simulate dispersal of the lineages, ignoring coalescence and mutation. Then, for each value of $\rho$ and $\mu$, we calculate the expected homozygosity and coalescence time distribution for each simulated trajectory. We then average over many independent trajectories. A major advantage of this two part method is that the same dispersal simulations can be used for calculating the homozygosity and coalescence time distribution for multiple choices of $\rho$ and $\mu$. Another advantage is that the second part of the method, in which conditional expectations are calculated for previously generated paths, is entirely deterministic. This reduces computational costs and noise in the estimations.

We simulate lineage motion using a discrete time random walk,

$$
X_{t+1}=X_{t}+\Delta X_{t}
$$

where $X_{t}$ represents the signed distance between two lineages at a given time (ignoring coalescence., i.e., assuming $\rho \rightarrow \infty$ ), and the step size, $\Delta X_{t}$, is a real valued random variable drawn from the dispersal distribution at each integer time $t$. For dispersal, we primarily use Lévy alpha-stable distributions, so $\Delta X_{t}$ has distribution:

$$
K(y)=\frac{1}{2 \pi} \int_{-\infty}^{\infty} d k \exp \left(-i k y-2 D_{\alpha}|k|^{\alpha}\right)
$$

Note that this differs from (1) because $\Delta t=1$, and there is an extra factor of two because $\Delta X_{t}$ is the sum of the two lineages' independent jumps, i.e., $K$ is the convolution of $K_{1}$ with itself. To simulate steeper tails with $\alpha>2$, we use an F-distribution, defined below in (15). We use the GNU Scientific Library's efficient pseudorandom generators for both stable distributions and the F-distribution (Galassi et al. 2009); because these are available only for the one-dimensional distributions, all our simulations are in one dimension.

For each simulated trajectory $\left\{x_{t}\right\}$, we then compute the conditional distribution of coalescence times $p\left(t \mid\left\{x_{t^{\prime} \leq t}\right\}\right)$ and conditional mean homozygosity $\psi\left(x \mid\left\{x_{t^{\prime}} \leq t\right\}\right)$ :

54

$$
\begin{aligned}
p\left(t \geq 1 \mid\left\{x_{t^{\prime} \leq t}\right\}\right) & =\left(1-e^{-\frac{1}{\rho} R\left(x_{t}\right)}\right) \exp \left[-\frac{1}{\rho} \sum_{t^{\prime}=1}^{t-1} R\left(x_{t^{\prime}}\right)\right], \\
\psi\left(x \mid\left\{x_{t^{\prime} \leq t}\right\}\right) & =\sum_{t=1}^{\infty} p\left(t \mid\left\{x_{t^{\prime} \leq t}\right\}\right) e^{-2 \mu t} .
\end{aligned}
$$

We start (13) and (14) at $t=1$ because we assume that the individuals are sampled immediately after dispersal, so no coalescence takes place at $t=0 . R(x)$ in (13) is a rectangular function representing a uniform rate of coalescence of all lineages within a distance $\delta$ :

$$
R(x) \equiv \begin{cases}\frac{1}{2 \delta} & \text { if }|x|<\delta \\ 0 & \text { otherwise. }\end{cases}
$$

For every time-step the lineages spend in this region, there is a probability of coalescence $1-e^{-\frac{1}{2 \rho \delta}}$. We discuss issues with the microscopic interpretation of this model after we introduce our analytical model below.

To obtain the unconditioned values $p(t \mid x)$ and $\psi(x)$, we then average (13) and (14) across all simulated trajectories. All error bars in plots show $68 \%$ confidence intervals, as determined by the percentile bootstrap with 10,000 bootstrap samples (Davison and Hinkley 1997). At large distances, the distribution of the probability of identity across sample trajectories is highly skewed, with most trajectories having very low probabilities of identity, but a few having the lineages rapidly jump close to each other and having a high probability of identity. This means that we cannot quantify the uncertainty in our estimates using, for example, the standard error of the mean, but it also means that we must simulate many independent trajectories to get good enough coverage for the bootstrap to be accurate (Chernick 2011).

We set $\delta=0.5$ for all simulations. For the simulations of mean homozygosity $\psi$ shown in Fig. 4 and Fig. 6, we simulate 250,000 independent runs of 1000 generations each for each combination of initial separation $x$ and tail parameter $\alpha$. We set the dispersal constant $D_{\alpha}$ indirectly by setting the characteristic spread $c$ of two lineages after one generation $(t=1), c=\left(2 D_{\alpha}\right)^{1 / \alpha}$, to be fixed at $c=250$ for $\alpha<2$, and $c=179.68$ for $\alpha=2.05$ (see below for the definition of $c$ for $\alpha>2$ ). For the largest initial separations, $x=e^{10}$ and $e^{11}$, coalescence within 1000 generations is very rare, so we increase the number of runs to $1.25 \times 10^{6}$. For Fig. 5 and Fig. 9, we choose $D_{\alpha}$ such that $c=0.2$, and simulate 10,000 independent runs of length 1000 generations each.

For the simulations of the cumulative distribution of coalescence times $P(t)$ shown in Fig. 8, we set initial separation $x=0$ and generate 10,000 independent trajectories of 1.5 million generations each for each combination of $c$ and tail parameter $\alpha$. We set $c=3.59$ for $\alpha>2, c=5$ for $1<\alpha<2$, and $c=1$ for $\alpha \leq 1$.

\section{Dispersal kernel for $\alpha>2$}

To simulate dispersal kernels with tail exponents $\alpha>2$, we draw $\Delta X_{t}$ from a Fisher F-distribution:

$$
K(y)=\frac{\Gamma(2 \alpha)}{\Gamma^{2}(\alpha) \omega}(y / \omega)^{\alpha-1}(1+(y / \omega))^{-2 \alpha} .
$$

At long times, the displacement distribution approaches that of a diffusive kernel, with dispersal constant equal to half the mean squared single-generation displacement of one lineage:

$$
D=\frac{c^{2}}{2}=\left(\frac{\alpha(2 \alpha-1)}{4(\alpha-2)(\alpha-1)^{2}}+\frac{\alpha^{2}}{4(\alpha-1)^{2}}\right) \omega^{2} .
$$




\section{Analytical model in one dimension}

\section{Generic dispersal}

We want to find a tractable analytical approximation to the model described above. For recurrent motion, the lineages will sometimes be in exactly the same place, and we can model coalescence with a $\delta$ distribution, i.e., as taking place at rate $\frac{1}{\rho} \delta\left(X_{t}\right)$. For transient motion, however, they will never coincide (Palyulin et al. 2014), and we must allow coalescence to take place at a finite distance. Let the coalescence kernel be some normalized distribution $\mathcal{N}(x)$ symmetric about $x=0$ and with width $\sim \delta$, with coalescence taking place at rate $\frac{1}{\rho} \mathcal{N}\left(X_{t}\right)$. The $\delta$-distribution is just the limit of $\mathcal{N}$ as $\delta$ goes to 0 , so we can treat the two cases together. Forien (2019) avoids this issue by using a spatial $\Lambda$-Fleming-Viot model, in which dispersal and coalescence are produced by the same long-range events, but we wish to keep long-range dispersal as a distinct process from short-range coalescence. As mentioned in the Simulation Model section, this creates issues with the microscopic interpretation of the model, which we discuss below in "Breakdown of models at small scales".

The coalescence time distribution is then:

$$
p(t \mid x)=E\left[\frac{1}{\rho} \mathcal{N}\left(X_{t}\right) \exp \left(-\frac{1}{\rho} \int_{0}^{t} d \tau \mathcal{N}\left(X_{\tau}\right)\right) \mid X_{0}=x\right],
$$

and the probability of identity is its Laplace transform:

$$
\psi(x)=\int_{0}^{\infty} d t p(t \mid x) e^{-2 \mu t} .
$$

There are several different ways to derive an explicit expression for $\psi$ from (17), including balancing mutation, coalescence, and dispersal over an infinitesimal time step (Malécot 1975; Barton et al. 2002) or, for Lévy flights, using a fractional diffusion equation (Janakiraman 2017) (see Appendix). Here we start with a generalization of Barton and Wilson (1995)'s expression for $p(t \mid x)$ that is valid for any two-lineage dispersal kernel $K$, not necessarily a Lévy flight, assuming that $\mathcal{N}(x)=\delta(x)$ :

$$
p(t \mid x)=\frac{1}{\rho} K(x \mid t)-\int_{0}^{t} d t^{\prime} p\left(t-t^{\prime} \mid 0\right) \frac{1}{\rho} K\left(x \mid t^{\prime}\right) .
$$

To interpret (18), notice that the first term is the probably of coalescing at time $t$ neglecting the possibility that the lineages have coalesced more recently. The second term corrects for these more recent coalescences: for every trajectory where the lineages coincide at $t^{\prime}<t$, we subtract off the probability that the lineages would coalesce at $t^{\prime}$ and then again exactly at $t$. Notice that we do not need to correct again for lineages that coincide three times, at $t^{\prime \prime}<t^{\prime}<t$ : the factor of $p$ guarantees that each trajectory is weighted appropriately.

We can immediately find a simple expression for $\psi$ for recurrent dispersal by taking the Laplace transform of (18):

$$
\psi(x)=\frac{1-\psi(0)}{\rho} \widetilde{K}(x, 2 \mu),
$$

where tilde denotes the Laplace transform. Plugging in $x=0$, we can solve (19) for $\psi(0)$ and express $\psi(x)$ purely in terms of the dispersal kernel:

$$
\psi(x)=\frac{\widetilde{K}(x, 2 \mu)}{\rho+\widetilde{K}(0,2 \mu)} .
$$

Note that, for diffusive dispersal, (19) reduces to the classical Wright-Malécot formula for isolation by distance (Barton et al. 2002).

For transient dispersal, we must consider a coalescence kernel of finite width, and (18) generalizes to:

$$
\begin{aligned}
p(t \mid x)= & \int d y \frac{1}{\rho} \mathcal{N}(y) K(x-y \mid t) \\
& -\int_{0}^{t} d t^{\prime} \int d y p\left(t-t^{\prime} \mid y\right) \frac{1}{\rho} \mathcal{N}(y) K\left(x-y \mid t^{\prime}\right) .
\end{aligned}
$$

(21) is exactly the same as (18) except that now we must integrate over possible locations $y$ of coalescence at both $t$ and $t^{\prime}$. Taking the Laplace transform of (21) now gives:

$$
\psi(x)=\frac{1}{\rho} \int d y(1-\psi(y)) \mathcal{N}(y) \widetilde{K}(x-y, 2 \mu) .
$$

To simplify (22), we can make the ansatz that $1-\psi(y)$ is nearly constant over all separations $|y| \lesssim \delta$ where $\mathcal{N}(y)$ is nonnegligible, allowing us to pull it out of the integral:

$$
\psi(x) \approx \frac{1-\psi(0)}{\rho} \int d y \mathcal{N}(y) \widetilde{K}(x-y, 2 \mu) .
$$

This ansatz will necessarily be accurate when identity is low, $\psi(0) \ll 1$ because $1-\psi$ will be close to 1 for all $y$. However, for $1-\psi(0) \ll 1$, the approximation can become inaccurate; we discuss this below. At long distances $x \gg \delta, \widetilde{K}$ will also be roughly constant in the integral, and we simply recover (19), although now only as an approximation:

$$
\psi(x \gg \delta) \approx \frac{1-\psi(0)}{\rho} \widetilde{K}(x, 2 \mu) .
$$

We see that the details of the short-range behavior only affect the long-range probability of identity by descent through the overall factor $1-\psi(0)$ (Barton et al. 2002). Mathematically, the main challenge is to find simple expressions for $\psi(0)$ and especially $\widetilde{K}$.

Because (24) is invalid for $x=0$, we cannot solve it directly for $\psi(0)$ as we could with (19), and so we must also work with (23). We can simplify the convolution in (23) by taking the spatial Fourier transform $\mathcal{F}\{\cdot\}$ :

$$
\widehat{\psi}(k) \approx \frac{1-\psi(0)}{\rho} \widehat{\mathcal{N}}(k) \widehat{\widetilde{K}}(k, 2 \mu) .
$$

where $\widehat{\psi}$ and $\widehat{\widetilde{K}}$ are the Fourier transforms of $\psi$ and $\widetilde{K}$.

\section{Lévy flight dispersal}

For Lévy flights, the characteristic function is $\widehat{K}(k \mid t)=$ $\exp \left(-2 D_{\alpha} t|k|^{\alpha}\right)$ and the Fourier-Laplace transform is $\widehat{\widetilde{K}}(k, 2 \mu)=$ $1 /\left(2 \mu+2 D_{\alpha}|k|^{\alpha}\right)$. (25) for $\widehat{\psi}$ is correspondingly simple:

$$
\frac{\widehat{\psi}(k)}{1-\psi(0)} \approx \frac{\widehat{\mathcal{N}}(k)}{2 \rho\left(\mu+D_{\alpha}|k|^{\alpha}\right)} .
$$

To get an explicit expression for $\psi$, we need to specify a form for the coalescence kernel $\mathcal{N}$. We will use a normal distribution with standard deviation $\delta$, which has the simple Fourier transform $\widehat{\mathcal{N}}(k)=\exp \left(-\delta^{2} k^{2} / 2\right)$. Then we can invert the Fourier transform in (26):

$$
\frac{\psi(x)}{1-\psi(0)} \approx \frac{1}{2 \pi \rho} \int_{0}^{\infty} d k \frac{\cos (k x) e^{-\delta^{2} k^{2} / 2}}{\mu+D_{\alpha} k^{\alpha}},
$$


which can be re-expressed in dimensionless units as

$$
\frac{\psi(x)}{1-\psi(0)} \approx \frac{1}{2 \pi \rho \mu \bar{x}} \int_{0}^{\infty} d \kappa \frac{\cos (\kappa x / \bar{x}) e^{-(\delta / \bar{x})^{2} \kappa^{2} / 2}}{1+\kappa^{\alpha}} .
$$

Examining (27), we see that the power law tail in the integrand can be cut off either when oscillations in the cosine factor become rapid at $k \sim 1 / x$ or by the normal factor at $k \sim 1 / \delta$. As long as we are sampling pairs that are outside the immediate range of coalescence, $x \gg \delta$, the former cutoff will happen at lower $k$, and therefore the normal factor can be neglected, leaving (in dimensionless form):

$$
\frac{\psi(x \gg \delta)}{1-\psi(0)} \approx \frac{1}{2 \pi \rho \mu \bar{x}} \int_{0}^{\infty} d \kappa \frac{\cos (\kappa x / \bar{x})}{1+\kappa^{\alpha}} .
$$

(29) can equivalently be derived directly from (24) by substituting in the Lévy flight dispersal kernel and writing $\widetilde{K}$ as the inverse Fourier transform of $\widehat{\widetilde{K}}$.

We can solve (28) for $\psi(x)$ by first evaluating it at $x=0$ to find $\psi(0)$; we do this below. But it is interesting that the ratio $\Psi(x) \equiv \psi(x) /(1-\psi(0))$ has the simplest relationship to the underlying parameters, as shown by Rousset (1997) for shortrange dispersal. $\Psi$ is closely related to Rousset (2000)'s statistic $a_{r}: a_{r}=\Psi(0)-\Psi(r)$. It is also related to the expected pairwise $F_{\mathrm{ST}}$ between demes separated by $x$ :

$$
E\left[F_{\mathrm{ST}}(x)\right]=\frac{\Psi(0)-\Psi(x)}{2+\Psi(0)-\Psi(x)} .
$$

\section{Probability of identity for distant pairs $x \gg \bar{x}, \alpha<2$}

For large $x \gg\left(D_{\alpha} t\right)^{1 / \alpha}$, the dispersal kernel has a simple asymptotic form for $\alpha<2$ (Nolan (2018), Theorem 1.12):

$$
K\left(x \gg\left(D_{\alpha} t\right)^{1 / \alpha} \mid t\right) \approx \frac{2 \Gamma(\alpha+1)}{\pi} \sin \left(\frac{\pi \alpha}{2}\right) \frac{D_{\alpha} t}{x^{\alpha+1}} .
$$

Plugging this into (24) and evaluating the Laplace transform gives (4), which was originally found by Nagylaki (1976):

$$
\frac{\psi(x \gg \bar{x})}{1-\psi(0)} \approx \frac{\Gamma(\alpha+1)}{2 \pi} \sin \left(\frac{\pi \alpha}{2}\right) \frac{D_{\alpha}}{\rho \mu^{2} x^{\alpha+1}} .
$$

\section{Probability of identity for distant pairs $x \gg \bar{x}, \alpha>2$}

There is no stable distribution with $\alpha>2$, but in discrete-time models such as the one we use in our simulations, we can consider single-generation jump kernels $K(y \mid 1)$ with power-law tails with $\alpha>2$. These will approach a diffusion with diffusion constant $D=\operatorname{Var}(K) / 2$. At long distances $y \gg \sqrt{D t}$, however, the tail will still be dominated by the probability of taking a single large jump (Vezzani et al. 2019), so for $x \gg \bar{x}$, we will have $K(x \mid t \lesssim 1 / \mu) \approx K(x \mid 1) t$. Plugging this into (24) and evaluating the Laplace transform gives:

$$
\frac{\psi(x \gg \bar{x})}{1-\psi(0)} \approx \frac{K(x \mid 1)}{4 \rho \mu^{2}} .
$$

For the F-distribution kernel (15) used in the simulations, this is

$$
\frac{\psi(x \gg \bar{x})}{1-\psi(0)} \approx \frac{\Gamma(2 \alpha)}{4 \Gamma^{2}(\alpha)} \frac{D_{\alpha}}{\rho \mu^{2} x^{\alpha+1}},
$$

where we have defined $D_{\alpha} \equiv \omega^{\alpha} /$ generation. (31) is confirmed by simulations (Fig. 6). We can then use the classic diffusive expression for $\psi(0)$ to get an explicit expression for probability of identity at large distances:

$$
\psi(x \gg \bar{x}) \approx\left(1+\frac{1}{4 \rho \bar{x} \mu}\right)^{-1} \frac{\Gamma(2 \alpha)}{4 \Gamma^{2}(\alpha)} \frac{D_{\alpha}}{\rho \mu^{2} x^{\alpha+1}} .
$$

Moderately long-range dispersal, $1<\alpha<2$

For $\alpha>1,(19)$ and (29) are exact for all $x$. Evaluating (29) for $x=0$ gives $\psi(0)$ :

$$
\psi(0)=\frac{1}{2 \alpha \sin (\pi / \alpha) \rho \bar{x} \mu+1} .
$$

Plugging (33) into (30) lets us solve for $\psi(x)$ at large distances $x \gg \bar{x}$ :

$$
\psi(x \gg \bar{x}) \approx \frac{\sin (\pi \alpha / 2) \Gamma(\alpha+1) /(2 \pi)}{1+1 /(2 \alpha \sin (\pi / \alpha) \rho \bar{x} \mu)} \frac{D_{\alpha}}{\rho \mu^{2} x^{\alpha+1}} .
$$

For $0<x \ll \bar{x}$, Janakiraman (2017) (Eq. (C1)) found that to leading order $\psi$ falls off as:

$$
\psi(x \ll \bar{x}) \approx \psi(0)\left[1-\frac{\alpha \sin (\pi / \alpha)}{\Gamma(\alpha) \cos (\pi(1-\alpha / 2))}\left(\frac{x}{\bar{x}}\right)^{\alpha-1}\right] .
$$

When $\alpha=2$, the above expression is equivalent to the classic diffusive result (3) for $x \ll \bar{x}$, which can be found by integrating (22) with $\delta=0$.

\section{Very long-range dispersal, $\alpha<1$}

For $\alpha<1$, the finite width $\delta$ of the coalescence kernel is important for determining $\psi(0)$. Dropping the cosine factor in (28) gives:

$$
\begin{aligned}
\frac{\psi(0)}{1-\psi(0)} & \approx \frac{1}{2 \pi \rho \mu \bar{x}} \int_{0}^{\infty} d \kappa \frac{e^{-(\delta / \bar{x})^{2} \kappa^{2} / 2}}{1+\kappa^{\alpha}} \\
& \approx \frac{\Gamma(1 / 2-\alpha / 2)}{2^{(\alpha+3) / 2} \rho D_{\alpha} \delta^{1-\alpha}}
\end{aligned}
$$

where in evaluating the integral we have assumed that $\delta \ll$ $\bar{x}$, i.e., that the mutation rate is not extremely large. We see that on small scales, the probability of identity by descent is independent of the mutation rate, i.e., there is a large probability that individuals from the same deme are differentiated even for infinitesimal mutation rates:

$$
\psi(0) \approx 1 /\left[1+\frac{2^{(\alpha+3) / 2} \pi}{\Gamma(1 / 2-\alpha / 2)} \rho D_{\alpha} \delta^{1-\alpha}\right]
$$

Very long-range dispersal of nearby lineages causes them to quickly wander away from each other, and for infinite range size many pairs will never coalesce. While (36) is only accurate for $\psi(0) \ll 1$, the independence from mutation rate should persist even for large $\psi(0)$.

Plugging (36) for $\psi(0)$ into (30) gives an explicit expression for the probability of identity of distant pairs with $x \gg \bar{x}$ :

$\psi(x \gg \bar{x}) \approx \frac{\Gamma(\alpha+1) \sin (\pi \alpha / 2) /(2 \pi)}{1+\Gamma(1 / 2-\alpha / 2) /\left(2^{(\alpha+3) / 2} \pi \rho D_{\alpha} \delta^{1-\alpha}\right)} \frac{D_{\alpha}}{\rho \mu^{2} x^{\alpha+1}}$.

For pairs that are nearby but still well outside of coalescence range, $\delta \ll x \ll \bar{x}$, the integral in (29) is dominated by $\kappa \gg 1$ and is approximately:

$$
\frac{\psi(\delta \ll x \ll \bar{x})}{1-\psi(0)} \approx \frac{\Gamma(1-\alpha) \sin (\pi \alpha / 2)}{2 \pi} \frac{x^{\alpha-1}}{\rho D_{\alpha}} .
$$

Again, the probability of identity is independent of the mutation rate to lowest order. Substituting in (36) gives an explicit expression for $\psi$ :

$\psi(\delta \ll x \ll \bar{x}) \approx \frac{\Gamma(1-\alpha) \sin (\pi \alpha / 2) /(2 \pi)}{1+\Gamma(1 / 2-\alpha / 2) /\left(2^{(\alpha+3) / 2} \pi \rho D_{\alpha} \delta^{1-\alpha}\right)} \frac{x^{\alpha-1}}{\rho D_{\alpha}}$. 


\section{Marginal case $\alpha=1$}

The analysis of the marginal case $\alpha=1$ is essentially the same as for $\alpha<1$ above, but we have separated it out because the form of the final expressions is very different. As with $\alpha<1$, the finite coalescence width $\delta$ is important for $x=0$ :

$$
\begin{aligned}
\frac{\psi(0)}{1-\psi(0)} & \approx \frac{1}{2 \pi \rho D_{1}} \int_{0}^{\infty} d \kappa \frac{e^{-(\delta / \bar{x})^{2} \kappa^{2} / 2}}{1+\kappa} \\
& =\frac{2 \ln (\bar{x} / \delta)+\ln 2-\gamma}{4 \pi \rho D_{1}},
\end{aligned}
$$

where $\gamma \approx 0.58$ is Euler's constant. Again assuming $\delta \ll \bar{x}=$ $D_{1} / \mu$, the constant terms in the numerator can be neglected and $\psi$ is approximately:

$$
\psi(0) \approx\left(1+\frac{2 \pi \rho D_{1}}{\ln (\bar{x} / \delta)}\right)^{-1} .
$$

Recall that the ansatz we used to derive (38) is only justified when $\psi(0) \ll 1$.

For pairs that are nearby but still well outside of coalescence range, $\delta \ll x \ll \bar{x}$, (29) gives:

$$
\frac{\psi(\delta \ll x \ll \bar{x})}{1-\psi(0)} \approx \frac{\ln (\bar{x} / x)-\gamma}{2 \pi \rho D_{1}} .
$$

Plugging the expression (38) for $\psi(0)$ into (39) and (30) gives explicit expressions for $\psi(x)$ at both short and long distances:

$$
\begin{aligned}
\psi(\delta \ll x \ll \bar{x}) & \approx \frac{\ln (\bar{x} / x)}{2 \pi \rho D_{1}+\ln (\bar{x} / \delta)} \\
\psi(x \gg \bar{x}) & \approx \frac{1}{2 \pi \rho D_{1}+\ln (\bar{x} / \delta)}\left(\frac{\bar{x}}{x}\right)^{2} .
\end{aligned}
$$

\section{Analytical model in two dimensions}

\section{Generic dispersal}

For generic dispersal, the solution for $\psi$ in two dimensions can again be found from (22), now with the integral over two spatial dimensions. The Fourier transform $\widehat{\psi}$ has the same form as the one-dimensional equation (25):

$$
\widehat{\psi}(k) \approx \frac{1-\psi(0)}{\rho} \widehat{\mathcal{N}}(k) \widehat{\widetilde{K}}(k, 2 \mu),
$$

where again we make the ansatz that $1-\psi(x)$ is approximately constant over the $x$ values where $\mathcal{N}(x)$ is non-negligible. This is again accurate for $\psi(0) \ll 1$, but may need to be adjusted for $1-\psi(0) \ll 1$. While (42) looks exactly like the one dimensional expression (25), its interpretation is different: $k$ is now the radial coordinate in two-dimensional $k$-space, and if we want to transform back to real space, we must use the two-dimensional inverse Fourier transform. For pairs that are far outside coalescence range, $x \gg \delta$, the simple relation (24) between $\psi(x)$ and $\widetilde{K}(x, 2 \mu)$ still holds.

\section{Lévy flight dispersal}

For a two-dimensional Lévy flight, the dispersal kernel takes the form of an isotropic stable distribution (Zolotarev 1981):

$$
K(y \mid t)=\frac{1}{2 \pi} \int_{0}^{\infty} d k k J_{0}(k y) \exp \left(-2 D_{\alpha} t k^{\alpha}\right),
$$

where $K(y \mid t)$ is the probability density of being at a particular point a distance $y$ away from the position at time 0 , and then gives: for $\psi(0) \ll 1$. $\delta \ll x \ll \bar{x}$ $\psi$ from (24):

$J_{0}$ is the zeroth Bessel function of the first kind. (43) is the two-dimensional inverse Fourier transform (equivalently, the inverse zeroth-order Hankel transform) of the characteristic function $\widehat{K}(k \mid t)=\exp \left(-2 D_{\alpha} t k^{\alpha}\right)$. The Fourier-Laplace transform is again $\widehat{\widetilde{K}}(x, 2 \mu)=1 /\left(2 \mu+2 D_{\alpha}|k|^{\alpha}\right)$. At large distances, $y \gg\left(D_{\alpha} t\right)^{1 / \alpha}, K$ has a power-law tail (Nolan 2013):

$$
K\left(y \gg\left(D_{\alpha} t\right)^{1 / \alpha} \mid t\right) \approx \frac{\alpha^{2} \Gamma(\alpha / 2)^{2}}{2^{1-\alpha} \pi^{2}} \sin \left(\frac{\pi \alpha}{2}\right) \frac{D_{\alpha} t}{x^{\alpha+2}} .
$$

In two dimensions, we must allow coalescence to take place at a finite distance for all $\alpha$ (Mörters and Peres 2010). For the coalescence kernel, we use an isotropic normal distribution $\mathcal{N}(x)$ with mean zero and standard deviation $\delta$, with coalescence taking place at rate $\frac{1}{\rho} \mathcal{N}\left(X_{t}\right)$. Inverting the Fourier transform in (42)

$$
\begin{aligned}
\frac{\psi(x)}{1-\psi(0)} & \approx \frac{1}{4 \pi \rho} \int_{0}^{\infty} d k \frac{k J_{0}(k x) e^{-\delta^{2} k^{2} / 2}}{\mu+D_{\alpha} k^{\alpha}} \\
& =\frac{1}{4 \pi \rho \mu \bar{x}^{2}} \int_{0}^{\infty} d \kappa \frac{\kappa J_{0}(\kappa x / \bar{x}) e^{-(\delta / \bar{x})^{2} \kappa^{2} / 2}}{1+\kappa^{\alpha}},
\end{aligned}
$$

The analysis of (46) parallels that of the one-dimensional case, but all $\alpha<2$ can be treated together for all distances $x$, not just $x \gg \bar{x}$, and so we can conduct one unified analysis moving from short distances to long ones.

Probability of identity for co-located pairs, $x=0$

For pairs sampled from the same location, $x=0$, the Bessel function in (46) is simply equal to one and can be dropped:

$$
\begin{aligned}
\frac{\psi(0)}{1-\psi(0)} & \approx \frac{1}{4 \pi \rho \mu \bar{x}^{2}} \int_{0}^{\infty} d \kappa \frac{\kappa e^{-(\delta / \bar{x})^{2} \kappa^{2} / 2}}{1+\kappa^{\alpha}} \\
& \approx \frac{\Gamma(1-\alpha / 2)}{2^{2+\alpha / 2} \pi \rho D_{\alpha} \delta^{2-\alpha}},
\end{aligned}
$$

where in the last line we have assumed that $\delta \ll \bar{x}$. Intuitively, (47) can be understood as roughly the ratio between the time to coalesce, i.e., the neighborhood size $\sim \rho \delta^{2}$ and the time $\sim$ $\delta^{\alpha} / D_{\alpha}$ that the lineages will spend in the same neighborhood before jumping apart. Note that mutation does not enter: in two dimensions, all $\alpha<2$ act like $\alpha<1$ does in one dimension, where locally mutation is irrelevant. Again, (47) is only accurate

Solving (47) for $\psi$ gives:

$$
\psi(0) \approx\left(1+\frac{2^{2+\alpha / 2} \pi}{\Gamma(1-\alpha / 2)} \rho D_{\alpha} \delta^{2-\alpha}\right)^{-1} .
$$

For $\alpha=2$, integrating (46) with $x=0$ recovers the classic diffusive result in two dimensions, which we expect to hold for pairs in contact when $\alpha \geq 2$ (Barton et al. 2002):

$$
\psi(0) \approx \frac{\ln (\bar{x} / \delta)}{\ln (\bar{x} / \delta)+4 \pi \rho D_{2}} .
$$

Probability of identity for separated but nearby pairs,

For pairs that are outside coalescence range, $x \gg \delta$, we can find

$$
\frac{\psi(\delta \ll x \ll \bar{x})}{1-\psi(0)} \approx \frac{1}{4 \pi \rho \mu \bar{x}^{2}} \int_{0}^{\infty} d \kappa \frac{\kappa J_{0}(\kappa x / \bar{x})}{1+\kappa^{\alpha}} .
$$


This looks different from the one-dimensional equation (27) because now we had to apply the two-dimensional inverse Fourier transform to $\widehat{\widetilde{K}}$ to obtain $\widetilde{K}$. For nearby pairs $x \ll \bar{x}$, the integral in (50) is dominated by $\kappa \gg 1$ and for $\alpha<2$ we can approximate the denominator in the integrand as $1+\kappa^{\alpha} \approx \kappa^{\alpha}$, giving:

$$
\frac{\psi(\delta \ll x \ll \bar{x})}{1-\psi(0)} \approx \frac{\Gamma(1-\alpha / 2)}{\Gamma(\alpha / 2) 2^{\alpha} \pi \rho D_{\alpha}} x^{\alpha-2} .
$$

The convergence of (50) to (51) is however quite slow in $x / \bar{x}$ when $\alpha$ is close to 0 or 2 . For example, for $x / \bar{x}=0.01$, the two expressions differ by $\approx 30-40 \%$ for $\alpha=0.25$ and $\alpha=$ 1.75 , and only approach to within $10 \%$ of each other at extreme values of $x / \bar{x}\left(\approx 10^{-5}\right.$ and $\approx 10^{-4}$ for $\alpha=0.25$ and $\alpha=1.75$, respectively).

Plugging (48) for $\psi(0)$ into (51) lets us solve for $\psi$ :

$\psi(\delta \ll x \ll \bar{x}) \approx\left(1+\frac{\Gamma(1-\alpha / 2)}{2^{2+\alpha / 2} \pi \rho D_{\alpha} \delta^{2-\alpha}}\right)^{-1} \frac{\Gamma(1-\alpha / 2)}{\Gamma(\alpha / 2) 2^{\alpha} \pi} \frac{x^{\alpha-2}}{\rho D_{\alpha}}$.

We see that in two dimensions, relatedness at short distances is independent of $\mu$ to leading order for all $\alpha<2$. However, the slow convergence mentioned above means that for most biologically reasonable parameter values, this should be interpreted as meaning that the dependence on mutation rate is weak rather than negligible.

For $\alpha=1$ and $\delta \ll x \ll \bar{x}$ we recover Eq. (A6) of Chave and Leigh Jr (2002) for Cauchy dispersal. Note that they consider distances large compared to the typical single-generation dispersal distance, $c \equiv\left(2 D_{\alpha}\right)^{1 / \alpha}$, but small compared to $\bar{x}$, and thus our result for $\delta \ll x \ll \bar{x}$ is consistent with their findings.

For $\alpha=2$ and $\delta \ll x \ll \bar{x}$ we can recover the known result for diffusive motion by approximating (46) as

$$
\frac{\psi(\delta \ll x \ll \bar{x})}{1-\psi(0)} \approx \frac{1}{4 \pi \rho \mu \bar{x}^{2}} \int_{0}^{\infty} d \kappa \frac{J_{0}(\kappa x / \bar{x}) \kappa}{1+\kappa^{2}} .
$$

Integrating (53) confirms that we find logarithmic scaling of $\psi(x)$ at short distances (Barton et al. 2002):

$$
\frac{\psi(x)}{1-\psi(0)} \approx \frac{1}{4 \pi \rho D_{2}} \ln (\bar{x} / x),
$$

which we expect to hold at $\delta \ll x \ll \bar{x}$ for all $\alpha \geq 2$.

\section{Probability of identity by descent for distant pairs, $x \gg \bar{x}$}

The probability of identity by descent for distant pairs $x \gg \bar{x} \gg$ $\delta$ can be immediately be read off from (24) by substituting in the tail of the two-dimensional dispersal kernel (44) for $\alpha<2$ :

$$
\frac{\psi(x \gg \bar{x})}{1-\psi(0)} \approx \frac{\alpha^{2} \Gamma(\alpha / 2)^{2}}{2^{3-\alpha} \pi^{2}} \sin \left(\frac{\pi \alpha}{2}\right) \frac{D_{\alpha}}{\rho \mu^{2}} x^{-\alpha-2} .
$$

Plugging in (48) for $\psi(0)$ lets us solve for $\psi$ :

$$
\psi(x \gg \bar{x}) \approx \frac{\alpha^{2} \Gamma(\alpha / 2) /\left[2^{3-\alpha} \pi \Gamma(1-\alpha / 2)\right]}{1+\Gamma(1-\alpha / 2) /\left[2^{2+\alpha / 2} \pi \rho D_{\alpha} \delta^{2-\alpha}\right]} \frac{D_{\alpha} x^{-\alpha-2}}{\rho \mu^{2}} .
$$

When $\alpha=2$, we instead recover classic expression for two dimensional diffusive motion at large distances (Barton et al. 2002):

$$
\frac{\psi(x \gg \bar{x})}{1-\psi(0)} \approx \frac{1}{4 \rho D_{2}} \frac{\exp (-x / \bar{x})}{\sqrt{2 \pi x / \bar{x}}}
$$

\section{Coalescence time distribution}

In this section we will find asymptotic expressions for the coalescence time distribution. As stated in the Results, intuitively we can think of the probability of identity $\psi$ as measuring the probability of the pair of lineages coalescing $\lesssim 1 /(2 \mu)$ generations ago. We can make this statement more rigorous using the Hardy-Littlewood tauberian theorem connecting the long (short) time probability of coalescence to the small (large) mutation rate limit of $\psi$. It states that a function $f(t)$ has the limiting behavior $f(t) \rightarrow \frac{1}{\Gamma(\beta)} t^{\beta-1} L(t)$ as $t \rightarrow \infty(t \rightarrow 0)$, where $L$ is a slowly varying function and $\beta>0$, if and only if its Laplace transform $\widetilde{f}(2 \mu)$ has the limiting behavior $\widetilde{f}(2 \mu) \rightarrow(2 \mu)^{-\beta} L(1 /(2 \mu))$ as $\mu \rightarrow 0(\mu \rightarrow \infty)$ (Feller (1971), XIII.5, Theorem 4).

\section{Recent times}

First we will consider the limit of recent times, $t \rightarrow 0 / \mu \rightarrow \infty$. For pairs sampled within coalescence range, $x \lesssim \delta$, by definition the coalescence time distribution approaches $p(t \mid x) \sim 1 /\left(\rho \delta^{d}\right)$, up to numerical factors that depend on the details of the coalescence kernel. Here $d$ is the dimensionality of the range, $d=1$ or 2. For pairs sampled well outside coalescence range, $x \gg \delta$, we can assume that $x \gg \bar{x}$ as well, since $\bar{x}=\left(D_{\alpha} / \mu\right)^{1 / \alpha} \rightarrow 0$ as $\mu \rightarrow \infty$. We can also assume that $1-\psi(0) \rightarrow 1$ is independent of $\mu$ to leading order. (For $\alpha<d$ our expressions for $\psi(0)$ (38) and (48) are also independent of $\mu$ and non-zero, but these are only valid when $\bar{x} \gg \delta$, i.e., when $\mu$ is not arbitrarily large.) We can therefore apply the tauberian theorem to (30) and (55) to obtain:

$$
\begin{aligned}
p\left(t \ll x^{\alpha} / D_{\alpha} \mid x\right) & \approx 2 d^{\alpha}\left(\frac{\Gamma(1+\alpha / d)}{\pi}\right)^{d} \sin \left(\frac{\pi \alpha}{2}\right) \frac{D_{\alpha} t}{\rho x^{\alpha+d}} \\
& \approx \frac{1}{\rho} K(x \mid t) .
\end{aligned}
$$

Our heuristic derivation in the Results section essentially proceeded in the opposite direction, starting from $p(t \ll$ $\left.x^{\alpha} / D_{\alpha} \mid x\right) \approx \frac{1}{\rho} K(x \mid t)$ and then deriving $\psi(x)$ from that. (57) is thus essentially just a restatement of our expressions for the tail of $\psi$, and its accuracy can be seen from the same simulation results shown in Fig. 4 and Fig. 6.

\section{Long times}

While there is a single unified expression for $p$ in the $t \rightarrow 0$ limit, corresponding to the single expression for $\psi$ in the $x \rightarrow \infty$ limit, for the opposite limit, $t \rightarrow \infty / \mu \rightarrow 0$, we must treat different values of $\alpha$ separately, just as we did for $\psi$ at small $x$. We verify our results with simulations, shown in Fig. 8.

For $\alpha<d$, we can simply take the inverse Laplace and Fourier transforms of (25) to find $p$, because $1-\psi(0)$ is independent of $\mu$ to leading order. Since we are concerned with times long compared to the time for the lineages to traverse the coalescence zone, $t \gg \delta^{\alpha} / D_{\alpha}$, the normal factor in (25) can be neglected and $p(t \mid x)$ is simply given by the inverse Laplace transform of (24):

$$
\begin{aligned}
p\left(t \gg \delta^{\alpha} / D_{\alpha} \mid x\right) & \approx \frac{1-\psi(0)}{\rho} K(x \mid t) \\
& \approx \frac{1-\psi(0)}{\alpha(2 \pi)^{d-1} \rho} \frac{\Gamma(d / \alpha)}{\left(2 D_{\alpha} t\right)^{d / \alpha}} \text { for } t \gg \frac{x^{\alpha}}{D_{\alpha}} .
\end{aligned}
$$

Integrating (59) yields the cumulative distribution for $t \gg 92$ 


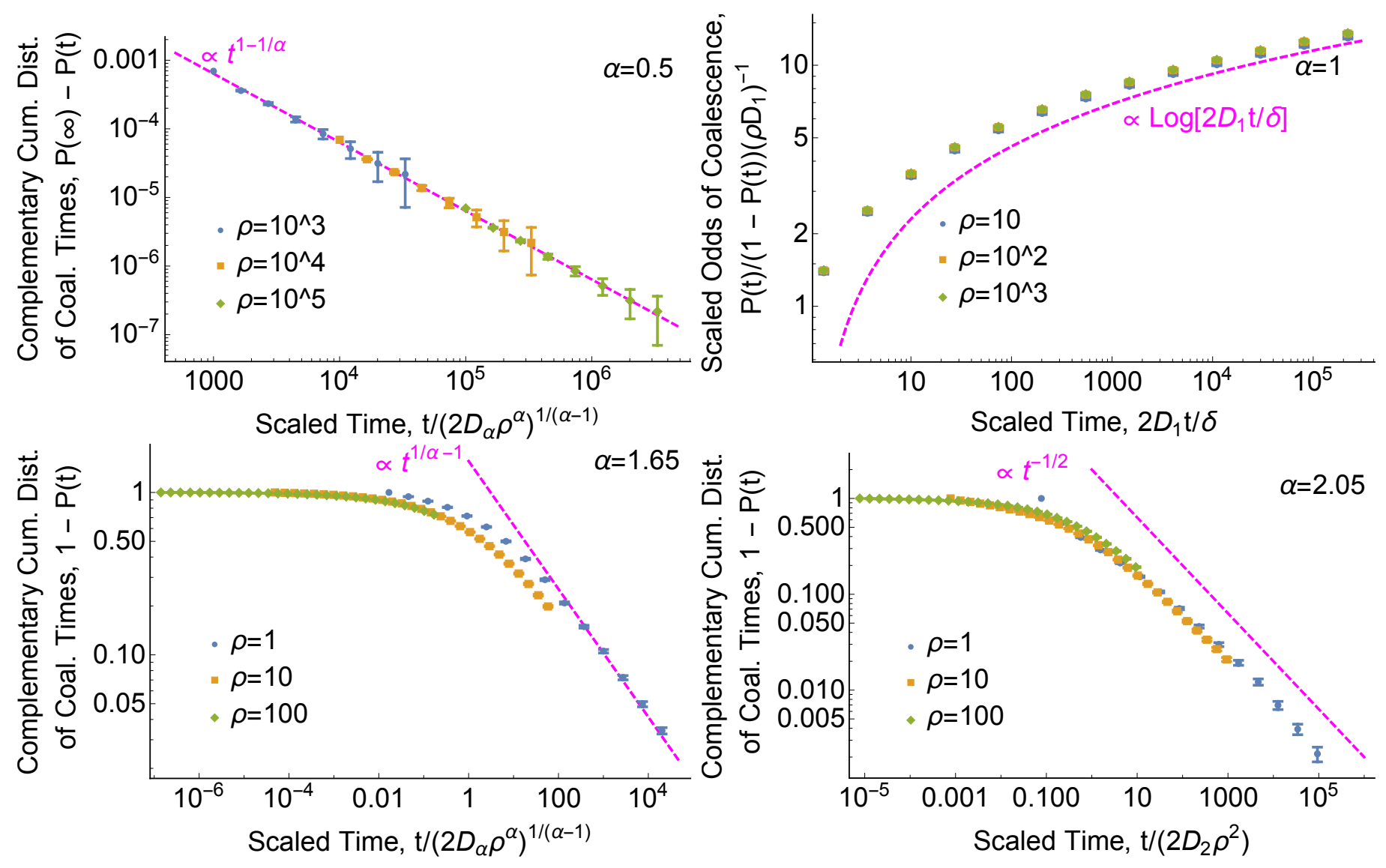

Figure 8 The distribution of coalescence times has a power law tail. Points show simulation results. Dashed magenta curves show the asymptotic predictions (in order of increasing $\alpha$ ) (60), (61), (63), and (65). Time is scaled to dimensionless units. See Simulation Methods section for $D_{\alpha}$ values. We show statistics based on the cumulative distribution $P(t)$ rather than the density $p(t)$ because simulation estimates for the latter are very noisy. Top left: for $\alpha<1$, the distribution of coalescence times is proportional to the probability of lineages being nearby, $K(0 \mid t) \propto \boldsymbol{t}^{1-1 / \alpha}$. Plot shows $P(\infty)-P(t)$ rather than $1-P(t)$ because lineages can disperse infinitely far away from each other and avoid coalescing entirely, i.e., $P(\infty)<1$. We use the simulated value of $P\left(t=10^{6}\right)$ to approximate $P(\infty)$. This empirical value deviates from the continuous-time prediction $(7)$ by $\approx 30 \%$ due to differences in the amount of coalescence in the first few generations (see "Breakdown of models at small scales"). Top right: the distribution of coalescence times has a logarithmic tail for $\alpha=1$. In this marginal case, lineages do eventually coalesce even in infinite ranges, but can take extremely long to do so. Bottom left: for $1<\alpha<2$, the distribution of coalescence times decays more quickly than the probability of lineages being nearby. The coalescence time distribution has a power-law tail, $p(t \mid x) \propto t^{1 / \alpha-2}$. This deviation from the scaling of the dispersal kernel at long times is due to the high probability of previous coalescence events. Bottom right: for $\alpha>2$, the coalescence time distribution may approach the diffusive limit. The scaling of $1-P$ appears to be close to that of the diffusive prediction, (65), but there is at least a difference in prefactor, perhaps again due to different probabilities of coalescence at very recent times. 
$x^{\alpha} / D_{\alpha}$

$P\left(t \gg \frac{x^{\alpha}}{D_{\alpha}} \mid x \gg \delta\right) \approx P(\infty \mid x)-\frac{\Gamma(d / \alpha)(1-\psi(0))\left(2 D_{\alpha}\right)^{-d / \alpha}}{(d-\alpha)(2 \pi)^{d-1} \rho t^{d / \alpha-1}}$,

where $P(\infty \mid x)=\lim _{\mu \rightarrow 0} \psi(x)$ is given by (7), (37), (48), or (52), depending on $x$ and $d$.

For the marginal case $\alpha=d$, we can use a slightly different statement of the tauberian theorem that applies for $\beta=0$ (Feller (1971), XIII.5, Theorem 2) to convert (38), (40), (49), and (54) to expressions for the cumulative distribution $P$ :

$$
P\left(t \gg x / D_{d} \mid x\right) \approx \begin{cases}{\left[1+\frac{2 d \pi \rho D_{d}}{\ln \left(2 D_{d} t / \delta\right)}\right]^{-1}} & \text { for } x \ll \delta \\ \frac{\ln \left(2 D_{d} t / x\right)}{2 d \pi \rho D_{d}+\ln \left(2 D_{d} t / \delta\right)} & \text { for } x \gg \delta .\end{cases}
$$

We can then differentiate to find the density $p$ :

$$
\begin{aligned}
p\left(t \gg \frac{x}{D_{d}} \mid x\right) \approx & \frac{2 d \pi \rho D_{d}}{t\left[2 d \pi \rho D_{d}+\ln \left(2 D_{d} t / \delta\right)\right]^{2}} \\
& \times \begin{cases}1 & \text { for } x \ll \delta \\
1+\frac{\ln (x / \delta)}{2 d \pi \rho D_{d}} & \text { for } x \gg \delta .\end{cases}
\end{aligned}
$$

Note that in two dimensions, $\alpha=d$ represents the diffusive limit, and we expect these expressions for the marginal case to hold for all $\alpha \geq 2$. lative distribution is trivial, $\lim _{\mu \rightarrow 0} \psi(x)=\lim _{t \rightarrow \infty} P(t \mid x)=1$, we must instead consider the complementary cumulative distribution, $\bar{P}(t \mid x) \equiv 1-P(t \mid x)$. Its Laplace transform is:

$$
\begin{aligned}
\widetilde{\bar{P}}(2 \mu) & =\frac{1}{2 \mu}-\widetilde{P}(2 \mu \mid x) \\
& =\frac{1}{2 \mu}[1-\psi(x)] .
\end{aligned}
$$

We can now apply the tauberian theorem to $\bar{P}$ and $\widetilde{\bar{P}}$. Since we
For $d=1<\alpha \leq 2$, since the leading behavior of the cumuare taking the $\mu \rightarrow 0$ limit, we have $\bar{x} \rightarrow \infty$, and we need only consider $\psi(x \ll \bar{x})$. Inspecting (33) and (35), we see that they have the limit:

$$
\frac{1}{2 \mu}[P(\infty \mid x)-\psi(x)] \rightarrow \alpha \sin (\pi / \alpha) \rho \bar{x} \text { as } \mu \rightarrow 0 .
$$

Since $\bar{x}=\left(D_{\alpha} / \mu\right)^{1 / \alpha}, \bar{P}$ has the limit:

$$
\bar{P}\left(t \gg x^{\alpha} / D_{\alpha} \mid x\right) \approx \alpha \sin \left(\frac{\pi}{\alpha}\right) \frac{\rho\left(2 D_{\alpha}\right)^{1 / \alpha}}{t^{1-1 / \alpha}} .
$$

Differentiating (63) yields the density $p(t \mid x)$ :

$$
p\left(t \gg x^{\alpha} / D_{\alpha} \mid x\right) \approx(\alpha-1) \sin \left(\frac{\pi}{\alpha}\right) \frac{\rho\left(2 D_{\alpha}\right)^{1 / \alpha}}{t^{2-1 / \alpha}},
$$

in agreement with Janakiraman (2017)'s Eq. 19.

For $\alpha=2$, (63) and (64) simplify to the classic diffusive results:

$$
\begin{aligned}
& \bar{P}\left(t \gg x^{2} / D \mid x\right) \approx 2 \rho \sqrt{2 D / t}, \\
& p\left(t \gg x^{2} / D \mid x\right) \approx \rho \sqrt{2 D / t^{3}} .
\end{aligned}
$$

For $\alpha>2$, we expect the coalescence rate $p(t \mid x) / \bar{P}(t \mid x)$ to behave similarly at long times, since the dispersal approaches a diffusion. But the distribution $P$ may be different, due to differences in the probability of early coalescence (Fig. 8, bottom right).

\section{Breakdown of models at small scales}

Great care must be taken in defining coalescent models in continuous space in order to guarantee that they have a consistent forward-time biological interpretation (Felsenstein 1975; Barton et al. 2010). We have not done this, and therefore the microscopic behavior of our models does not correspond to any biological population. However, the behavior at large scales (time long compared to one generation, distance long compared to the coalescence scale $\delta$ and the typical single-generation dispersal distance $c \equiv\left(2 D_{\alpha}\right)^{1 / \alpha}$ ) should still be realistic (Barton et al. 2002). Forien (2019) has recently given an example of a biologically consistent forward-time, continuous-space model that we believe would be described by our results on macroscopic scales. We also believe that our results would describe a stepping-stone model of discrete demes of size $\sim \rho \delta^{d}$ separated by distance $\sim \delta$.

The key place in which the microscopic details matter even for large distances and long times is the factor $1-\psi(0)$ which appears in many of our expressions. As discussed above, for $\alpha<$ $d$ even here the microscopic details are not necessarily important, but for $\alpha \geq d$ they are. Practically speaking, this quantity would typically have to simply be measured in a population or else treated as a fitting parameter when matching the large-scale predictions to data.

At a microscopic level, we expect that our continuous-time analytic model should deviate from discrete-time models such as the one we use in our simulations. As shown in Fig. 9, this becomes apparent for $\alpha<1$ in one dimension (or more generally, $\alpha<d)$. The two differ at scales smaller than the typical singlegeneration dispersal distance, $x<c=\left(2 D_{\alpha}\right)^{1 / \alpha}$, when this scale is large compared to the coalescence scale, $c \gg \delta$. In continuous time, nearby pairs with $x \ll c$ would be able to coalesce at times smaller than a single generation, $t \ll 1$. But in discrete time no pairs can coalesce until $t=1$, by which time the dispersal kernel $K(x \mid 1)$ is roughly flat out to $x \lesssim c$, and probability of identity thus becomes approximately constant for $x \lesssim c$. (For $\alpha \geq d$, the continuous-time model already predicts that $\psi$ should be changing slowly at $x \ll \bar{x}$, and therefore we do not expect a disagreement with the discrete-time model.) Recall that our discrete-time model assumes no coalescence at $t=0$ even for lineages starting at $x<\delta$; if we were to change this, $\psi$ would discontinuously jump up to a second, higher plateau for $x<\delta$.

We can estimate the discrete-time value of $\psi(x \ll c)$ from a heuristic argument, at least when $\psi \ll 1$. In the absence of coalescence, the probability of the lineages being within coalescence range of each other in generation $t \geq 1$ is $\approx(2 \delta) K(x \mid t) \approx$ $(2 \delta) K(0 \mid t)$. For $\psi \ll 1$, including the possibility of coalescence will only slightly decrease this probability. Given that the lineages are in coalesce range, they coalesce with probability is $\approx K(0 \mid t) / \rho$ and we can find $\psi$ by summing over all generations:

$$
\begin{aligned}
\psi(x \ll c) & \approx \sum_{t=1}^{\infty} \frac{K(0 \mid t)}{\rho} \\
& =\frac{\Gamma(1 / \alpha) \zeta(1 / \alpha)}{\alpha} \frac{1}{\rho c}
\end{aligned}
$$

where $\zeta$ is the Riemann zeta function. Fig. 9 shows that (67) accurately describes the simulations. $1 /(2 \delta \rho)$. So in any one generation the probability of coalescence 


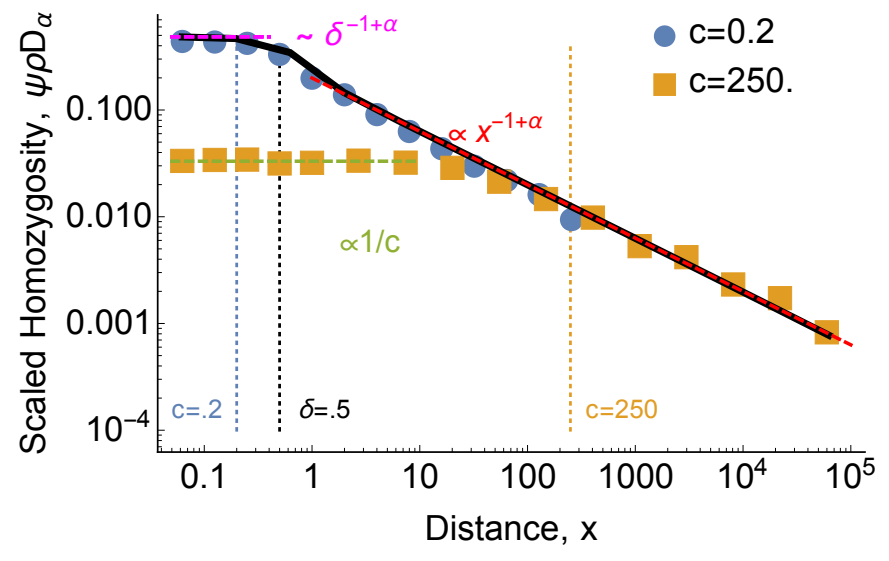

Figure 9 For very long-range dispersal, $\alpha<1$, continuoustime and discrete-time models differ at short distances.

Scaled probability of identity $\psi$ as a function of distance $x$ for $\alpha=0.5, \delta=0.5$, and $\rho=100$. Points show discrete-time simulation results. For the continuous-time model, the black curve shows the result of numerically integrating (27), while the dashed red and magenta lines show the asymptotic approximations (6) and (7), respectively. The continuous-time model predicts that $\psi$ should only plateau within the coalescence distance $\delta$, but for distance between $\delta$ and the typical singlegeneration dispersal distance $c$, the change in $\psi$ is driven by the probability of coalescing at $0<t \ll 1$. In the discrete-time model, these lineages have to wait until $t=1$ to coalesce, leading to a lower, broader plateau, given by (67) (dashed green line). This discrepancy only exists for $\delta<x \ll c$, i.e., if $c<\delta$ then the discrete-time and continuous-time models agree (blue points).

\section{Acknowledgments}

The authors thank Nick Barton, Reed Cartwright, Graham Coop, and David Field for helpful suggestions. DBW is partially supported by an Investigator award from the Simons Foundation. TBS is partially supported by NSF award 1806833 (PoLS SRN) and by a Woodruff Fellowship from Emory University.

\section{Literature Cited}

Adams, W., A. Griffin, and G. Moran, 1992 Using paternity analysis to measure effective pollen dispersal in plant populations. The American Naturalist 140: 762-780.

Aguillon, S. M., J. W. Fitzpatrick, R. Bowman, S. J. Schoech, A. G. Clark, et al., 2017 Deconstructing isolation-by-distance: the genomic consequences of limited dispersal. PLoS genetics 13: e1006911.

Al-Asadi, H., D. Petkova, M. Stephens, and J. Novembre, 2019 Estimating recent migration and population-size surfaces. PLoS genetics 15: e1007908.

Allman, B. E. and D. B. Weissman, 2018 Hitchhiking in space: Ancestry in adapting, spatially extended populations. Evolution 72: 722-734.

Atkinson, R., C. Rhodes, D. Macdonald, and R. Anderson, 2002 Scale-free dynamics in the movement patterns of jackals. Oikos 98: 134-140.

Austerlitz, F., C. W. Dick, C. Dutech, E. K. Klein, S. OddouMuratorio, et al., 2004 Using genetic markers to estimate the pollen dispersal curve. Molecular ecology 13: 937-954.

Bacles, C. F. and R. A. Ennos, 2008 Paternity analysis of pollenmediated gene flow for fraxinus excelsior 1 . in a chronically fragmented landscape. Heredity 101: 368-380.

Baguette, M., 2003 Long distance dispersal and landscape occupancy in a metapopulation of the cranberry fritillary butterfly. Ecography 26: 153-160.

Barton, N., 1983 Multilocus clines. Evolution pp. 454-471.

Barton, N. H., F. Depaulis, and A. M. Etheridge, 2002 Neutral evolution in spatially continuous populations. Theoretical population biology 61: 31-48.

Barton, N. H., A. M. Etheridge, J. Kelleher, and A. Véber, 2013 Genetic hitchhiking in spatially extended populations. Theoretical Population Biology 87: 75 - 89.

Barton, N. H. and G. M. Hewitt, 1985 Analysis of hybrid zones. Annual review of Ecology and Systematics 16: 113-148.

Barton, N. H., J. Kelleher, and A. M. Etheridge, 2010 A new model for extinction and recolonization in two dimensions: Quantifying phylogeography. Evolution 64: 2701 - 2715.

Barton, N. H. and I. Wilson, 1995 Genealogies and geography. Philosophical Transactions of the Royal Society of London. Series B: Biological Sciences 349: 49-59.

Battey, C. J., P. L. Ralph, and A. D. Kern, 2020 Space is the place: Effects of continuous spatial structure on analysis of population genetic data. Genetics 215: 193-214.

Bialozyt, R., B. Ziegenhagen, and R. Petit, 2006 Contrasting effects of long distance seed dispersal on genetic diversity during range expansion. Journal of evolutionary biology 19: 12-20.

Bradburd, G. S., G. M. Coop, and P. L. Ralph, 2018 Inferring continuous and discrete population genetic structure across space. Genetics 210: 33-52.

Bradburd, G. S. and P. L. Ralph, 2019 Spatial population genetics: It's about time. Annual Review of Ecology, Evolution, and Systematics 50: 427-449. 
Bradburd, G. S., P. L. Ralph, and G. M. Coop, 2016 A spatial framework for understanding population structure and admixture. PLoS genetics 12: e1005703.

Brockmann, D. and L. Hufnagel, 2007 Front propagation in reaction-superdiffusion dynamics: Taming Lévy flights with fluctuations. Physical review letters 98: 178301.

Brockmann, D., L. Hufnagel, and T. Geisel, 2006 The scaling laws of human travel. Nature 439: 462.

Carmi, S., L. Turgeman, and E. Barkai, 2010 On distributions of functionals of anomalous diffusion paths. Journal of Statistical Physics 141: 1071-1092.

Cayuela, H., Q. Rougemont, J. G. Prunier, J.-S. Moore, J. Clobert, et al., 2018 Demographic and genetic approaches to study dispersal in wild animal populations: A methodological review. Molecular ecology 27: 3976-4010.

Chave, J. and E. G. Leigh Jr, 2002 A spatially explicit neutral model of $\beta$-diversity in tropical forests. Theoretical population biology 62: 153-168.

Chen, X., Y. Hu, and J. Song, 2012 Feynman-Kac formula for fractional heat equation driven by fractional white noise. arXiv preprint arXiv:1203.0477 .

Chernick, M. R., 2011 Bootstrap methods: A guide for practitioners and researchers, volume 619. John Wiley \& Sons.

Clark, J. S., 1998 Why trees migrate so fast: confronting theory with dispersal biology and the paleorecord. The American Naturalist 152: 204-224.

Dai, X., G. Shannon, R. Slotow, B. Page, and K. J. Duffy, 2007 Short-duration daytime movements of a cow herd of African elephants. Journal of Mammalogy 88: 151-157.

Davison, A. C. and D. V. Hinkley, 1997 Bootstrap methods and their application, volume 1. Cambridge University Press.

Devaux, C., C. Lavigne, F. Austerlitz, and E. Klein, 2007 Modelling and estimating pollen movement in oilseed rape (brassica napus) at the landscape scale using genetic markers. Molecular Ecology 16: 487-499.

Fayard, J., E. K. Klein, and F. Lefèvre, 2009 Long distance dispersal and the fate of a gene from the colonization front. Journal of evolutionary biology 22: 2171-2182.

Feller, W., 1971 An introduction to probability theory and its applications, volume 2. Wiley, second edition.

Felsenstein, J., 1975 A pain in the torus: some difficulties with models of isolation by distance. The American Naturalist 109: 359-368.

Forien, R., 2019 Isolation by distance patterns arising from short range and long range dispersal-a forwards in time approach. arXiv preprint arXiv:1907.07930 .

Fric, Z. and M. Konvicka, 2007 Dispersal kernels of butterflies: power-law functions are invariant to marking frequency. Basic and Applied Ecology 8: 377-386.

Gagnaire, P.-A., T. Broquet, D. Aurelle, F. Viard, A. Souissi, et al., 2015 Using neutral, selected, and hitchhiker loci to assess connectivity of marine populations in the genomic era. Evolutionary applications 8: 769-786.

Galassi, M., J. Davies, J. Theiler, B. Gough, G. Jungman, et al., 2009 GNU Scientific Library Reference Manual. Network Theory Ltd., third edition.

Hallatschek, O. and D. S. Fisher, 2014 Acceleration of evolutionary spread by long-range dispersal. Proceedings of the National Academy of Sciences 111: E4911-E4919.

Harris, K. and R. Nielsen, 2013 Inferring demographic history from a spectrum of shared haplotype lengths. PLoS genetics 9.
Hey, J., 2010 Isolation with migration models for more than two populations. Molecular biology and evolution 27: 905-920.

Ibrahim, K. M., R. A. Nichols, and G. M. Hewitt, 1996 Spatial patterns of genetic variation generated by different forms of dispersal during range expansion. Heredity 77: 282-291.

Janakiraman, D., 2017 Lévy flights in the presence of a point sink of finite strength. Physical Review E 95: 012154.

Jespersen, S., R. Metzler, and H. C. Fogedby, 1999 Lévy flights in external force fields: Langevin and fractional Fokker-Planck equations and their solutions. Physical Review E 59: 2736.

Jones, A. G. and W. R. Ardren, 2003 Methods of parentage analysis in natural populations. Molecular ecology 12: 2511-2523.

Kimura, M. and G. H. Weiss, 1964 The stepping stone model of population structure and the decrease of genetic correlation with distance. Genetics 49: 561.

Koenig, W. D., D. Van Vuren, and P. N. Hooge, 1996 Detectability, philopatry, and the distribution of dispersal distances in vertebrates. Trends in ecology \& evolution 11: 514-517.

Kwaśnicki, M., 2017 Ten equivalent definitions of the fractional Laplace operator. Fractional Calculus and Applied Analysis 20: 7-51.

Lischke, A., G. Pang, M. Gulian, F. Song, C. Glusa, et al., 2020 What is the fractional Laplacian? a comparative review with new results. Journal of Computational Physics 404: 109009.

Lundgren, E. and P. L. Ralph, 2019 Are populations like a circuit? comparing isolation by resistance to a new coalescent-based method. Molecular ecology resources 19: 1388-1406.

Malécot, G., 1975 Heterozygosity and relationship in regularly subdivided populations. Theoretical population biology $\mathbf{8}$ : 212-241.

Mancinelli, R., D. Vergni, and A. Vulpiani, 2003 Front propagation in reactive systems with anomalous diffusion. Physica D: Nonlinear Phenomena 185: 175-195.

Maruyama, T., 1971 The rate of decrease of heterozygosity in a population occupying a circular or a linear habitat. Genetics 67: $437-454$.

Maruyama, T., 1972 Rate of decrease of genetic variability in a two-dimensional continuous population of finite size. Genetics 70: $639-651$.

Metzler, R., A. V. Chechkin, and J. Klafter, 2009 Lévy statistics and anomalous transport: Lévy flights and subdiffusion. Encyclopedia of Complexity and Systems Science pp. 5218-5239.

Metzler, R. and J. Klafter, 2000 The random walk's guide to anomalous diffusion: a fractional dynamics approach. Physics reports 339: 1-77.

Mörters, P. and Y. Peres, 2010 Brownian motion, volume 30. Cambridge University Press.

Nagylaki, T., 1976 The relation between distant individuals in geographically structured populations. Mathematical Biosciences 28: 73-80.

Nathan, R., G. Perry, J. T. Cronin, A. E. Strand, and M. L. Cain, 2003 Methods for estimating long-distance dispersal. Oikos 103: 261-273.

Nolan, J. P., 2013 Multivariate elliptically contoured stable distributions: theory and estimation. Computational Statistics 28: 2067-2089.

Nolan, J. P., 2018 Stable Distributions - Models for Heavy Tailed Data. Birkhäuser, Boston, In progress, Chapter 1 online at http:/ / fs2.american.edu/jpnolan/www/stable/stable.html.

Palyulin, V. V., A. V. Chechkin, and R. Metzler, 2014 Lévy flights do not always optimize random blind search for sparse targets. Proceedings of the National Academy of Sciences 111: 2931- 
2936.

Paulose, J. and O. Hallatschek, 2020 The impact of long-range dispersal on gene surfing. Proceedings of the National Academy of Sciences 117: 7584-7593.

Paulose, J., J. Hermisson, and O. Hallatschek, 2019 Spatial soft sweeps: patterns of adaptation in populations with long-range dispersal. PLoS genetics 15: e1007936.

Petkova, D., J. Novembre, and M. Stephens, 2016 Visualizing spatial population structure with estimated effective migration surfaces. Nature genetics 48: 94.

Ralph, P. and G. Coop, 2013 The geography of recent genetic ancestry across Europe. PLoS biology 11: e1001555.

Ree, R. H. and S. A. Smith, 2008 Maximum likelihood inference of geographic range evolution by dispersal, local extinction, and cladogenesis. Systematic biology 57: 4-14.

Rieux, A., T. Lenormand, J. Carlier, L. De Lapeyre de Bellaire, and V. Ravigné, 2013 Using neutral cline decay to estimate contemporary dispersal: a generic tool and its application to a major crop pathogen. Ecology letters 16: 721-730.

Ringbauer, H., G. Coop, and N. H. Barton, 2017 Inferring recent demography from isolation by distance of long shared sequence blocks. Genetics 205: 1335-1351.

Robledo-Arnuncio, J. and F. Rousset, 2010 Isolation by distance in a continuous population under stochastic demographic fluctuations. Journal of evolutionary biology 23: 53-71.

Robledo-Arnuncio, J. J., F. Austerlitz, and P. E. Smouse, 2006 A new method of estimating the pollen dispersal curve independently of effective density. Genetics 173: 1033-1045.

Rohlf, F. J. and G. D. Schnell, 1971 An investigation of the isolation-by-distance model. The American Naturalist 105: 295-324.

Rousset, F., 1997 Genetic differentiation and estimation of gene flow from F-statistics under isolation by distance. Genetics 145: 1219-1228.

Rousset, F., 2000 Genetic differentiation between individuals. J Evol Biol 13: 58-62.

Rousset, F. and R. Leblois, 2011 Likelihood-based inferences under isolation by distance: two-dimensional habitats and confidence intervals. Molecular biology and evolution 29: 957973.

Sanmartín, I., P. Van Der Mark, and F. Ronquist, 2008 Inferring dispersal: a bayesian approach to phylogeny-based island biogeography, with special reference to the canary islands. Journal of Biogeography 35: 428-449.

Slatkin, M., 1991 Inbreeding coefficients and coalescence times. Genetics Research 58: 167-175.

Slatkin, M., 1993 Isolation by distance in equilibrium and nonequilibrium populations. Evolution 47: 264-279.

Slatkin, M. and H. E. Arter, 1991 Spatial autocorrelation methods in population genetics. The American Naturalist 138: 499-517.

Slatkin, M. and N. H. Barton, 1989 A comparison of three indirect methods for estimating average levels of gene flow. Evolution 43: 1349-1368.

Sotka, E. E. and S. R. Palumbi, 2006 The use of genetic clines to estimate dispersal distances of marine larvae. Ecology 87: 1094-1103.

Vallaeys, V., R. C. Tyson, W. D. Lane, E. Deleersnijder, and E. Hanert, 2017 A lévy-flight diffusion model to predict transgenic pollen dispersal. Journal of the Royal Society Interface 14: 20160889.

Vezzani, A., E. Barkai, and R. Burioni, 2019 Single-big-jump principle in physical modeling. Physical Review E 100: 012108.
Wang, J. and A. W. Santure, 2009 Parentage and sibship inference from multilocus genotype data under polygamy. Genetics 181: 1579-1594.

Weissman, D. B. and O. Hallatschek, 2017 Minimal-assumption inference from population-genomic data. eLife 6: e24836.

Whitlock, M. C. and D. E. McCauley, 1999 Indirect measures of gene flow and migration: $F_{\mathrm{ST}} \neq 1 /(4 \mathrm{Nm}+1)$. Heredity 82 : 117-125.

Willson, M., 1993 Dispersal mode, seed shadows, and colonization patterns. In Frugivory and seed dispersal: ecological and evolutionary aspects, pp. 261-280, Springer.

Wingen, L. U., J. K. Brown, and M. W. Shaw, 2007 The population genetic structure of clonal organisms generated by exponentially bounded and fat-tailed dispersal. Genetics 177: 435-448.

Wright, S., 1946 Isolation by distance under diverse systems of mating. Genetics 31: 39.

Zaburdaev, V., S. Denisov, and J. Klafter, 2015 Lévy walks. Reviews of Modern Physics 87: 483.

Zolotarev, V. M., 1981 Integral transformations of distributions and estimates of parameters of multidimensional spherically symmetric stable laws. In Contributions to Probability: A Collection of Papers Dedicated to Eugene Lukacs, edited by J. Gani and V. K. Rohatgi, pp. 283 - 305, Academic Press.

\section{Appendix: Alternative derivations of the probability of identity by descent}

\section{Starting from a recursion equation}

(25) can also be derived starting from a recursion equation for $\psi$ requiring that it remain constant over an infinitesimal timestep dt (Malécot 1975; Barton et al. 2002):

$$
\psi(x)=\frac{d t}{\rho}(1-\psi(x)) \mathcal{N}(x)+e^{-2 \mu d t} \int d y \psi(x-y) K(y \mid d t) .
$$

(68) is saying that at equilibrium the local increase in identity due to coalescence (first term) must be balanced by the loss of identity due to mutation and the spreading of identity due to dispersal (both included in the second term).

Taking the spatial Fourier transform $\mathcal{F}\{\cdot\}$ of (68) simplifies the second term at the expense of complicating the first:

$$
\widehat{\psi}(k)=\frac{d t}{\rho} \mathcal{F}\{(1-\psi(x)) \mathcal{N}(x)\}(k)+e^{-2 \mu d t} \widehat{\psi}(k) \widehat{K}(k \mid d t) .
$$

Solving for $\widehat{\psi}$ gives:

$$
\begin{aligned}
\widehat{\psi}(k) & =\frac{\mathcal{F}\{(1-\psi(x)) \mathcal{N}(x)\}(k)}{\rho} \frac{d t}{1-e^{-2 \mu d t} \widehat{K}(k \mid d t)} \\
& =\frac{\mathcal{F}\{(1-\psi(x)) \mathcal{N}(x)\}(k)}{\rho} \sum_{j=0}^{\infty} d t e^{-2 \mu j d t} \widehat{K}(k \mid d t)^{j},
\end{aligned}
$$

where in the second line we can take the Taylor series expansion because $e^{-2 \mu d t}<1$ and $\widehat{K}(k \mid d t) \leq 1$ since it is a characteristic function. Assuming dispersal is Markovian, we can simplify (69) by noting that $\widehat{K}(k \mid d t)^{j}=\widehat{K}(k \mid j d t)$, i.e., the distribution after time $j d t$ is just the convolution of $j$ dispersal steps of time $d t$ each. Using this, we can convert the sum into an integral to find 
(25):

4 where in the second line we have used the same ansatz that

$51-\psi(x) \approx 1-\psi(0)$ for $|x| \lesssim \delta$ that we used in the main text.

\section{Fractional diffusion equation}

For Lévy flight dispersal, (26) can also be derived using a fractional diffusion equation. When $X_{t}$ follows a diffusion, (17) can be written as a Feynman-Kac (diffusion) equation for $\psi$ (Barton et al. 2002; Allman and Weissman 2018). For $\alpha<2$, this generalizes to a fractional differential equation:

$$
0=2 D_{\alpha}\left(\frac{\partial^{2}}{\partial x^{2}}\right)^{\alpha / 2} \psi(x)-2 \mu \psi(x)+\frac{1}{\rho} \mathcal{N}(x)(1-\psi(x)),
$$

where $\left(\frac{\partial^{2}}{\partial x^{2}}\right)^{\alpha / 2}$ is a Riesz fractional derivative, defined by its Fourier transform $\mathcal{F}\left\{\left(\frac{\partial^{2}}{\partial x^{2}}\right)^{\alpha / 2} f\right\}(k)=-|k|^{\alpha} \mathcal{F}\{f\}(k)$ (Metzler et al. 2009; Carmi et al. 2010; Janakiraman 2017). It is therefore simpler to consider the Fourier transform of (70), which is equivalent to (26):

$$
\begin{aligned}
0 & =-\left(2 D_{\alpha}|k|^{\alpha}+2 \mu\right) \widehat{\psi}(k)+\frac{1}{\rho} \mathcal{F}\{\mathcal{N}(x)(1-\psi(x))\}(k) \\
& \approx-\left(2 D_{\alpha}|k|^{\alpha}+2 \mu\right) \widehat{\psi}(k)+\frac{1-\psi(0)}{\rho} \widehat{\mathcal{N}}(k) .
\end{aligned}
$$

For all $\alpha<2$, the solution for $\psi$ in two dimensions can be also written as a fractional differential equation (Chen et al. 2012):

$$
0=2 D_{\alpha}\left(\frac{\partial^{2}}{\partial x^{2}}+\frac{1}{x} \frac{\partial}{\partial x}\right)^{\frac{\alpha}{2}} \psi(x)-2 \mu \psi(x)+\frac{1}{\rho} \mathcal{N}(x)(1-\psi(x)),
$$

where $\left(\frac{\partial^{2}}{\partial x^{2}}+\frac{1}{x} \frac{\partial}{\partial x}\right)^{\frac{\alpha}{2}}$ is a fractional Laplacian, defined by its Fourier transform $\mathcal{F}\left\{\left(\frac{\partial^{2}}{\partial x^{2}}+\frac{1}{x} \frac{\partial}{\partial x}\right)^{\alpha / 2}\right\}(k)=-|k|^{\alpha} \mathcal{F}\{f\}(k)$ (Kwaśnicki 2017; Lischke et al. 2020). Note that the rotational symmetry of the problem allows us to write the Laplacian in terms of just the radial coordinate $x$, and ignore the angular coordinate. The two-dimensional Fourier transform of (72) has exactly the same form as (71), although again the interpretation is different as $k$ is now the radial coordinate in two-dimensional $k$-space. 